\title{
Apprendre aux étudiants paramédicaux à collaborer : dynamique et continuum de pratiques collaboratives dans un dispositif de formation
}

\author{
SERVANE BOUJARD \\ Université de Rennes, Institut de Formation en Pédicurie-podologie, Ergothérapie et \\ Kinésithérapie (IFPEK), s.boujard@ifpek.org \\ YANN LE FAOU \\ Université de Rennes, Laboratoire CREAD-EA3875, Institut de Formation en Pédicurie- \\ podologie, Ergothérapie et Kinésithérapie (IFPEK), y.lefaou@ifpek.org \\ NICOLAS GUIRIMAND \\ Université de Rouen-Normandie, MCU, Laboratoire CIRNEF, UFR des Sciences et de la \\ Société, nicolas.guirmand@univ-rouen.fr
}

\section{Résumé}

L'évolution des besoins en santé nécessite, pour une population donnée, d'améliorer la collaboration entre les différents professionnels des domaines sanitaire et médico-social. Cela demande de repenser les formations initiales en santé pour permettre aux jeunes professionnels de s'adapter à ces nouvelles pratiques de terrain. C'est pourquoi, l'Institut de Formation en Pédicurie-podologie, Ergothérapie et Kinésithérapie (IFPEK) de Rennes a mis en place un dispositif de formation à la collaboration interprofessionnelle. La présente recherche analyse l'effet de ce dispositif sur la professionnalité émergente de 181 étudiants pédicure-podologues, ergothérapeutes et kinésithérapeutes, notamment dans leur capacité à mettre en place des interactions interprofessionnelles dans le cadre d'un travail collaboratif. Dix entretiens semidirectifs auprès de ces étudiants ainsi que des observations directes lors de la formation ont été menés. En s'appuyant sur le modèle canadien du « Continuum des pratiques de collaboration en santé et service sociaux », les résultats montrent qu'une grande majorité des étudiants s'orientent spontanément vers des pratiques collaboratives mais que leurs interactions sont parfois insuffisantes pour répondre aux problématiques de santé les plus complexes.

\footnotetext{
Abstract

Population health needs evolution entails a change towards more collaborative work between the different professional actors of the medico-social field. This requires a rethinking of the basic healthcare training programs to enable the young professionals to adapt for such new work practices. This is why the Pedicure-Podology, Occupational therapy and Physiotheray
} 
Institute (IFPEK) of Rennes has developed a training programme that promotes interprofessional collaboration. An evaluation of this training programme questions the future capacity of 181 students to implement pertinent and adaptive interactions between them in the context of collaborative work. Ten semi-structured interviews were carried with these students and observations was made during the training. Based on the «Continuum of interprofessional collaborative practice in health and social care », the results of this evaluation show that a majority of students spontaneously turn towards collaborative practices, but their interactions are sometimes insufficient to address the most complex health issues.

\section{Mots-clés}

Collaboration interprofessionnelle, étudiants, formation initiale, santé.

\section{Key words}

Interprofessional collaboration, students, initial training, health.

\section{Introduction}

L'évolution de l'état de santé en France conduit aujourd'hui les institutions et les professionnels à concevoir de manière différente les pratiques autour des usagers des soins. La loi du 13 août 2004, relative à l'assurance maladie, a mis en avant la notion de «parcours de soins » pour favoriser une continuité de l'accompagnement médico-social en préconisant une meilleure coordination et collaboration des professionnels médico-sociaux. Dans ce contexte de décloisonnement du système de santé, il s'agit d'orienter les formations initiales vers une culture du travail collaboratif interprofessionnel. C'est pourquoi, l'Institut de Formation en Pédicurie-podologie, Kinésithérapie et Ergothérapie (IFPEK) de Rennes, établissement d'enseignement supérieur dans le domaine de la santé, a souhaité intégrer dans le cursus de ses étudiants un apprentissage du travail interprofessionnel, les préparant à ces futures collaborations. De nombreuses initiatives ont vu le jour pour favoriser ces apprentissages. Des recherches ont d'ailleurs présenté l'intérêt porté par les étudiants pour ces formations: «l'intérêt de formations centrées sur la problématique de l'inter professionnalité, permettant de rencontrer les autres professionnels de santé, déconstruire les représentations et travailler dans une logique de collaboration inter professionnelle est souligné par les étudiants. » (Fiquet, Huge et Annezo, 2015, p. 115). L'objectif principal est de former des professionnels de terrain sensibilisés aux évolutions des besoins en santé et adaptables aux exigences organisationnelles des institutions. 


\section{Contexte de la recherche}

Trois journées pédagogiques, appelées «InterPEK », ont été conçues pour tenter de répondre aux besoins de formation à la collaboration interprofessionnelle au sein de l'IFPEK. Une évaluation diagnostique a démontré l'intérêt de regrouper les étudiants par niveaux d'études. Une journée de formation inter-filières a donc été programmée par promotion; soit une planification sur trois ans, impliquant un travail régulier et progressif. Des objectifs pédagogiques ont été définis pour chaque niveau. En première année, une journée de formation devrait permettre aux étudiants de construire des représentations partagées et ainsi favoriser l'ouverture aux autres. En $2^{\mathrm{e}}$ année, une seconde journée est organisée afin d'expérimenter et de comprendre la démarche interprofessionnelle autour d'une problématique de santé engageant une pratique collaborative. Enfin, en $3^{\mathrm{e}}$ année, une dernière journée permettrait de développer une réflexion sur le travail en réseau et la recherche au sein des professions de rééducation et de réadaptation.

Notre retour d'expérience, présenté ici, porte uniquement sur le dispositif réalisé pendant la deuxième année de formation. Dans ce dispositif, 181 étudiants ont participé. Trois grands groupes ont été constitués ; chacun assistant à la projection d'un témoignage vidéo exposant une problématique de santé complexe. Ces témoignages décrivent le parcours de santé des personnes et leurs difficultés rencontrées au quotidien. À la suite des visionnages, des groupes de huit à dix participants, composés des trois professions, ont eu comme consignes de proposer une évaluation en regard de la problématique de santé, en rapport avec le projet de vie de la personne et sur une thématique imposée : douleur, chute ou déplacement. L'évaluation est ici entendue comme la première étape dans l'élaboration collectif du diagnostic. Il s'agit d'un recueil de données permettant une première représentation de la situation de handicap de la personne. Le résultat du travail collaboratif devait être présenté sous forme de carte mentale. Les patients filmés ont été présents une partie de la journée pour permettre de répondre aux questions des étudiants et de compléter leur recueil de données. La mise en commun des cartes mentales par thématique et une restitution orale ont clôturé la journée. De nombreux échanges entre les formateurs, les patients et les étudiants ont permis d'engager une réflexion collective sur leur perception de l'atelier proposé au cours de cette journée. Ce scénario pédagogique a été choisi afin de saisir - au plus près de la réalité du terrain - l'importance d'une mise en condition lorsqu'il s'agit d'éducation interprofessionnelle dans les secteurs médico-sociaux (Gachoud, Gallant, Lucarelli et Oberhauser, 2017). La conception de ce dispositif a ainsi 
privilégié une démarche réflexive des étudiants dans un contexte se rapprochant le plus possible de la réalité des pratiques (Aiguier, Poirette et Pélissier, 2016).

Ainsi, le dispositif propose aux étudiants de confronter leurs connaissances cliniques aux exigences d'une situation favorisant une dynamique inter-individuelle d'échanges, par la création d'espaces de dialogue.

\section{Cadre théorique}

Pour éclairer les professionnels médico-sociaux sur la collaboration interprofessionnelle, le Réseau de Collaboration sur les Pratiques Interprofessionnelles (RCPI) en santé et services sociaux s'est engagé « dans un processus de réflexion et de construction avec des membres de la communauté universitaire (professeurs, chercheurs) et des établissements (gestionnaires, professionnels) ainsi que des personnes et de leurs proches afin de développer un outil de transfert des connaissances illustrant les plus récents travaux de conceptualisation de la collaboration interprofessionnelle. » (Careau, Brière, Houle, Dumont, Maziade, Paré, Desaulniers et Museux, 2014, p. 3). Le modèle ainsi créé permet à des professionnels de santé qui le souhaitent de se repérer sur un « continuum» de pratiques collaboratives interprofessionnelles. Il est composé de quatre axes distincts. Le premier, la situation, représente la problématique de santé de l'usager des soins et conditionne les trois autres. La complexification des besoins bio-psycho-sociaux de la personne entraîne une intention de collaboration ( $2^{\mathrm{e}}$ axe) de plus en plus partagée par les professionnels, des interactions plus intenses ( $3^{\mathrm{e}}$ axe) et une combinaison de savoirs disciplinaires ( $\left.4^{\mathrm{e}} \mathrm{axe}\right)$ au profit d'un seul savoir commun avec une co-construction de la compréhension de la situation. D'Amour, Sicotte et Levy (1999) montrent également l'importance d'un rapprochement des pensées des professionnels nécessaires dans leur travail en interdépendance autour des situations de santé rencontrées.

Il existe donc plusieurs types de pratiques collaboratives qui relèvent d'un jeu d'interactions, entre les acteurs concernés, dont la fréquence diffère selon les contextes rencontrés.

La première pratique est «la pratique en parallèle ». Elle met en relation au moins deux professionnels de disciplines différentes dans le but unique d'informer l'autre sur ses interventions appliquées à l'usager des soins concernés. Les interactions sont minimes voir absentes car il peut simplement s'agir de la lecture d'un rapport rédigé par un autre professionnel. Leur interdépendance est donc très faible. Cette pratique ne nécessite pas de coordination particulière si ce n'est la mise à disposition des outils de communication. Mourey 
appuie l'importance de la communication entre les professionnels de santé : «il existe d'une part un rôle propre du rééducateur fournissant des données émanant d'outils d'évaluation qui lui sont spécifiques, et d'autre part la nécessité de mettre en rapport ces informations avec celles émanant des autres évaluations médicales ou paramédicales. » (Mourey, 2005, p. 187).

La seconde pratique du continuum est «la pratique par consultation/référence » qui est liée à l'intention d'échanger de l'information avec au moins un autre professionnel d'un domaine différent. Cette collaboration a pour objectif de clarifier, de compléter ou d'orienter les interventions de chacun des professionnels. Les interactions demeurent peu nombreuses mais cette pratique implique une reconnaissance des limites de son expertise professionnelle et le recours à un autre professionnel. L'engagement entre les acteurs n'est pas réciproque et la prise en considération de l'information récoltée dépend du professionnel l'ayant demandée. Ainsi, les savoirs des autres champs disciplinaires, s'ils sont utilisés, sont simplement juxtaposés.

Le troisième type de pratique fait référence à «la pratique de concertation ». L'interaction y est modérée et bidirectionnelle mais il existe une interdépendance entre les professionnels. Leurs actions s'inscrivent dans un souci de cohérence et l'intention est d'organiser une offre de soins avec une vision globale de la personne concernée. Pour l'atteinte des objectifs, une coordination entre les acteurs sera mise en place pour assurer la complémentarité entre les savoirs et les compétences mis en jeu. Une planification des interventions est indispensable ainsi qu'un engagement réciproque des professionnels sollicités.

Enfin la quatrième et dernière pratique collaborative de ce continuum s'appuie sur le partage de «pratique de soins et de services ». Elle implique une prise de décision partagée et une harmonisation des points de vue par plusieurs professionnels de différentes disciplines. Les interactions sont plus intenses et nécessitent une forte cohésion, par un engagement formel de tous les acteurs. Henri et Lundgren-Cayrol (2001) précisent que «l'engagement se traduit par la participation active de tous les membres d'un groupe et par d'authentiques efforts pour réaliser les tâches et atteindre le but. »(p. 112). La pratique de soins et de services partagés exige également un partage des responsabilités et une bonne connaissance du domaine d'action des autres professions pour assurer une définition commune de la situation. Careau et al. (2014) qualifient ce type de pratique d'interdisciplinarité car elle exige un partage des savoirs (p. 16). Pour que les équipes travaillent efficacement, cette pratique requiert une bonne coordination. « Les équipes travaillent plus efficacement dans les conditions suivantes : objectifs clairs, bonne communication, bonne coordination [...]»(Oandasa et al., 2006, p. 1). 
La situation d'un usager des soins ayant des besoins peu complexes pourra facilement être résolue par une pratique en parallèle. Au contraire, si la situation est très complexe et pour une meilleure qualité des soins, les professionnels devront se diriger vers une collaboration de type pratique de soins et de services partagés.

\section{Problématique}

Dans les milieux de la rééducation, réadaptation et réhabilitation, en structure de soins ou en ambulatoire, lorsque les kinésithérapeutes, les ergothérapeutes et les pédicures-podologues se rencontrent, c'est que la situation de la personne concernée est complexe. Effectivement, ces trois professions réunies engagent une expertise multidimensionnelle autour d'une personne très souvent, dans une situation de forte dépendance pour les activités de vie quotidienne.

L'évolution des besoins en santé et, en conséquence, le changement des pratiques professionnelles tournées vers l'interprofessionnalité incite les étudiants à collaborer pour trouver des solutions de prise en soins par-delà les frontières de leur propre champ disciplinaire. Ils devraient être en mesure d'intégrer les champs de compétences des autres professionnels ou au moins réussir à s'y référer pour répondre aux problématiques de santé complexes auxquelles ils sont confrontés.

Sont-ils prêts à cela? Est-ce que le développement professionnel des étudiants peut leur permettre de construire des connaissances favorisant la collaboration interprofessionnelle? Dans un contexte de formation inter-filières pour des professions de rééducation, comment des pratiques de soins et de services partagés pourraient être envisagées ? Ces dernières étant conditionnées par l'intensité des interactions mises en jeu, les étudiants s'engageront-ils suffisamment? Réussiront-ils à communiquer et à se coordonner afin de poursuivre un objectif commun, voire à co-construire des connaissances transversales ? Intégreront-ils, dans leur professionnalité, les pratiques collaboratives pour faire face aux limites de leur propre expertise dans des situations complexes?

Ainsi, notre recherche interroge la professionnalité émergente (Jorro, 2011) des étudiants dans leur capacité à mettre en place des interactions appropriées dans le cadre d'un travail collaboratif. Son objectif principal est d'identifier comment le dispositif de formation mis en œuvre par l'IFPEK participe à la professionnalisation des étudiants dans le cadre de journée de collaboration interprofessionnelle impliquant des pratiques de soins et de services partagés. 


\section{Méthode de recueil des données}

Pour recueillir les données, plusieurs outils ont été utilisés. Dans un premier temps, cinq formateurs ont observé les étudiants pendant le déroulement du dispositif munis d'une grille d'observation construite sur la base du modèle de référence (Annexe 1). L'observation des cartes mentales nous a permis d'affiner l'analyse de nos résultats. Elles ont été catégorisées selon les composantes des pratiques collaboratives de soins et de services partagés (Annexe 3), pour pouvoir être corroborées aux réponses des étudiants recueillis lors des entretiens (Annexe 2) qui ont lieu quelques semaine après la journée d'étude.

Les données recueillies ici concernent des pratiques émergentes de collaboration de la part d'étudiants en formation initiale. Notre analyse vise une compréhension des actions mises en œuvre plutôt que la mesure de ces dernières. Les outils utilisés nous permettent de porter un regard sur le vécu de ces futurs professionnels et sur leur façon d'interagir en réponse à une problématique engageant la collaboration interprofessionnelle. Notre approche, basée sur le modèle du «Continuum des pratiques interprofessionnelles en santé et services sociaux » (Careau et al. 2014) met en évidence l'importance des interactions avec le milieu et les autres professionnels dans une réflexivité permanente de la part des étudiants amenant à la construction de nouvelles connaissances.

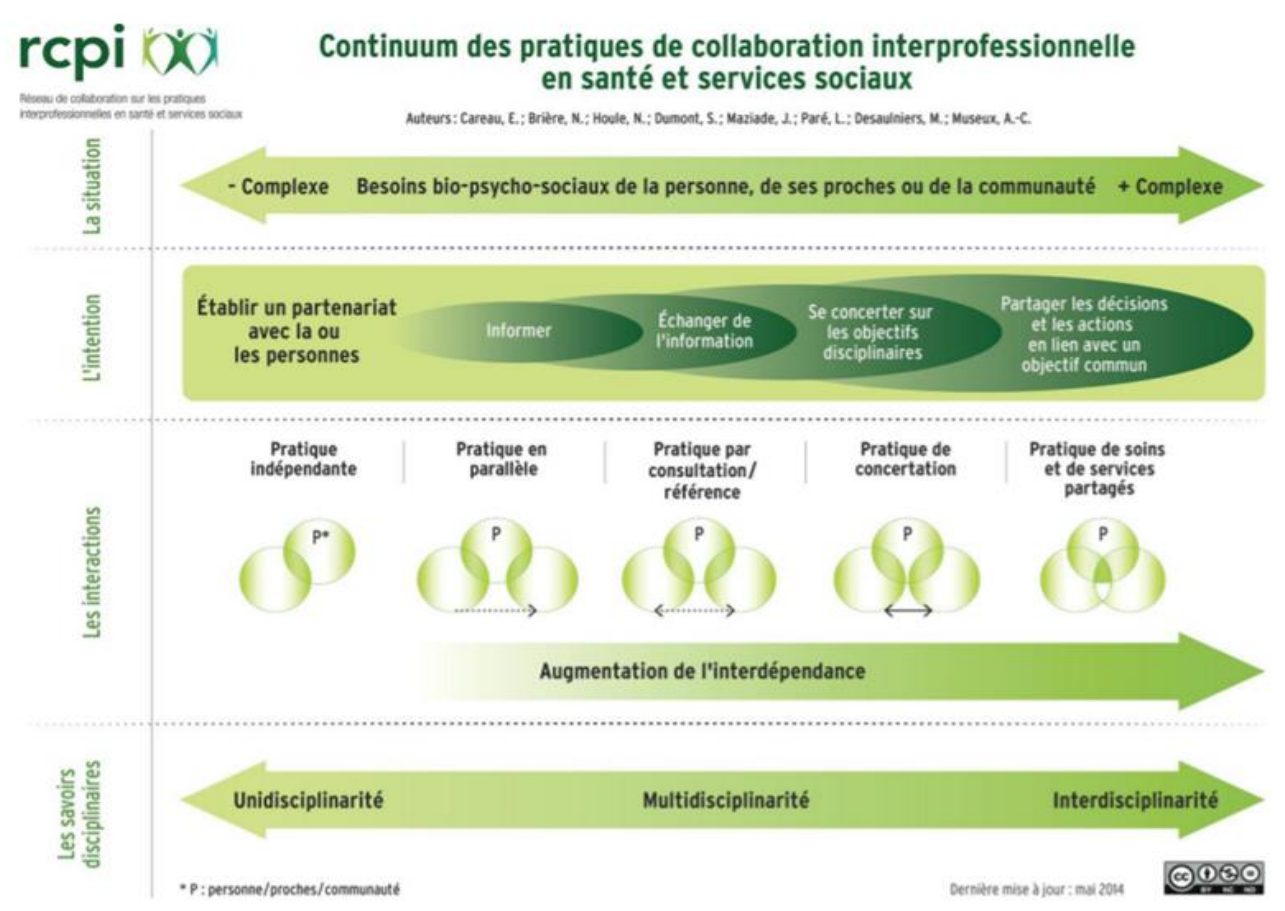

Figure 1 : Continuum des pratiques de collaboration interprofessionnelle en santé et services sociaux 


\section{Résultats}

Les résultats suivants cherchent à mettre en évidence si, dans le dispositif, les étudiants ont pu mettre en place une collaboration pertinente au regard du contexte de l'activité proposée.

\subsection{Interdépendance}

Le « continuum des pratiques de collaboration interprofessionnelle en santé et services sociaux » montre que plus la situation d'une personne est complexe plus l'interdépendance des professionnels médico-sociaux augmente. Cette interdépendance face à la situation de santé proposée est beaucoup évoquée par les étudiants interrogés. Lors des entretiens, huit d'entre eux expriment qu'il fallait, effectivement, être plusieurs professionnels de domaines différents pour répondre correctement aux besoins de la personne rencontrée au cours du travail collaboratif.

Cependant, cette proximité rendue nécessaire entre les étudiants, a parfois été compliquée à assumer. Le faible recul de ces jeunes professionnels leur permet difficilement de faire face aux réactions d'un patient qui, par exemple, met en avant une profession par rapport à une autre. Si l'intérêt des participants pour l'objet de la focalisation est détourné, la pratique de soins et de services partagés ne peut avoir lieu puisque, selon notre modèle, elle se caractérise par un engagement formel dans l'action collective. Mais nos entretiens ont montré qu'un seul étudiant sur dix était concerné, ce qui représente une minorité dans notre échantillon. La majorité d'entre eux a effectivement exprimé un fort niveau d'interdépendance entre les membres du groupe de travail, autour de la personne rencontrée, les incitant ainsi à entrer dans une relation formelle engageant une participation active.

\subsection{Engagement et participation active}

Les entretiens et les observations réalisés nous permettent de mettre en évidence un engagement formel dans une grande majorité des groupes inter-filières. Cependant deux groupes observés sur cinq n'ont pas réussi à établir une réciprocité dans leurs interactions. Nos analyses font ressortir une forme de détachement du travail collaboratif qui a pu être mise en évidence dans le discours d'une étudiante qui parle des membres de son groupe : «On trouvait qu'elles étaient avec leurs portables, donc ça n'était pas... On sentait bien qu'ils n'avaient pas trop envie d'être là ».

Nos observations directes ont également révélé des personnes inactives, en retrait du reste du groupe, exprimant une certitude de ne pas pouvoir s'engager, par un manque de connaissances 
et de capacités mobilisables dans la situation proposée. Ces étudiants sont restés centrés sur ces remises en questions et n'ont pas réussi à intégrer la collaboration indispensable au travail proposé.

Enfin, l'analyse des entretiens montre que la lutte de concurrence entre praticiens est bel et bien un enjeu notamment lorsqu'il s'agit de délimiter des territoires (juridictions) et des pratiques professionnelles.

\subsection{Cohésion pour des actions collectives inter-filières}

Les résultats révèlent, d'une part, une ouverture des étudiants aux autres par des présentations individuelles et professionnelles réciproques, d'autre part, une adaptation des pratiques pour la poursuite d'un but commun établi lors d'un consensus inter-filières. C'est bien la problématique de santé qui devient une problématique commune. On observe de ce fait, une forte cohésion entre étudiants, investis pour tenter de répondre à la demande, toutes filières confondues.

Cependant, les étudiants n'ayant pas répondu aux composantes d'engagement et d'interdépendance n'ont pas réussi à intégrer la cohésion créée au sein de leur groupe. Un groupe sur les cinq observés est particulièrement concerné ainsi que deux étudiants sur les dix interrogés.

\subsection{Partage des responsabilités}

L'analyse des entretiens a permis de mettre en évidence un partage solidaire des responsabilités entre les étudiants. Ils expriment une répartition des actions en fonction des spécificités liées aux filières et l'attribution de tâches plus transversales nécessaire à la cohérence de l'ensemble du travail.

Les résultats montrent également que la confiance et le respect mutuel dans la professionnalité des autres ont permis des actions réciproques coordonnées au sein des groupes inter-filières. Treize cartes mentales sur les 20 relevées montrent un ensemble cohérent, exprimant des actions inter-filières coordonnées et des évaluations personnalisées face à la problématique de santé rencontrée.

\section{5. Échange de savoirs disciplinaires}

Neuf étudiants entretenus sur dix et les cinq groupes observés ont montré une volonté d'aller rencontrer les autres cultures professionnelles, au travers d'interactions verbales, et de confronter leurs représentations afin de les faire évoluer vers de nouveaux apprentissages. Ce 
processus est fondamental dans les pratiques de soins et de services partagés puisqu'il ouvre les frontières tout en valorisant les savoirs propres à chacun. Le travail collaboratif des étudiants les a menés vers une production retranscrite sur papier. Onze cartes mentales témoignent d'une harmonisation des points de vues dans le but de répondre à la problématique de santé rencontrée.

\subsection{Vers des pratiques de soins et de services partagés}

Les pratiques de soins et de services partagés impliquent des interactions multiples, sollicitant la professionnalité entière des acteurs. En deuxième année de formation, la professionnalité émergente des étudiants ne leur permet qu'une maîtrise partielle de leurs connaissances et capacités à mobiliser en situation. De plus, leur expérience encore débutante entraîne une pratique timide lorsqu'il s'agit de les mettre en action. Nos entretiens révèlent que tous les étudiants ont, à un moment donné, rencontré des difficultés dans leurs interactions, risquant parfois la rupture de collaboration. Certains ont pourtant réussi à surmonter ces obstacles, dans le but de réaliser le travail qui était demandé, et surtout, de répondre à la demande d'une personne en situation de handicap. Pour ces futurs professionnels, la présence des composantes de la pratique collaborative de soins et de service partagés, dans leurs discours, dans leurs faceà-face et à travers leurs productions, montrent qu'ils sauront juger du type de pratique nécessaire dans une situation de collaboration en santé. Pour ceux-là, le dispositif pédagogique a répondu à l'objectif défini au départ, à savoir, expérimenter et comprendre la démarche interprofessionnelle autour d'une problématique de santé engageant une pratique collaborative. Pour d'autres, des limites ont été mises en évidence notamment à travers des postures de détachement ou encore de protection vis-à-vis d'une identité professionnelle encore fragile.

\section{Discussion}

On retrouve une hiérarchie de classement dans le discours des étudiants lorsqu'ils s'expriment sur les relations entre professionnels de santé. Cette « hiérarchie » entre professions existerait, selon eux, sous une forme pyramidale, renvoyant au modèle traditionnel des organisations médico-sociales. Ce modèle renvoie au pouvoir prescripteur du médecin et à la séparation des territoires professionnels (Dubar, 2015, p. 132). Les voies d'admission en formation dans le domaine de la santé sont considérées par les étudiants comme participant fortement à son installation et à sa pérennisation. Cependant, tous les étudiants ont, à un moment donné, démontré leur détachement par rapport à ces représentations. Lorsqu'ils évoquent leurs futures interactions entre professionnels de santé, cette « hiérarchie » est très critiquée. La collaboration est adoptée comme un moyen de mettre en œuvre des accompagnements de qualité auprès des 
usagers des soins. Ces conflits de valeurs entre représentations construites sur le vécu et leur projection montrent des tensions inhérentes au développement professionnel chez ces étudiants. Ces tensions ont pu représenter une limite dans la mise en place d'une pratique collaborative et les outils utilisés dans notre analyse n'ont pas pu en rendre compte.

Ensuite, nous avons pu observer que pour certains étudiants, trouver une place dans le processus de collaboration engagé au sein des groupes de travail, n'a pas été évident. Leur professionnalité émergente ne leur permettait peut-être pas encore de mobiliser toutes les connaissances nécessaires à l'exercice demandé. À ce sujet, Gachoud et al. (2017) soulignent que «pour pouvoir travailler en interprofessionnalité, il faut être clair sur sa profession, sur ce qu'elle apporte, au patient et à ses proches, d'unique et de différents des autres professions. » (p. 153). Ainsi, pour ces auteurs il demeure important de favoriser un «juste dosage » entre professionnalité et interprofessionnalité. Il semblerait également, que l'utilisation d'un outil commun, tel que la Classification Internationale du Fonctionnement, du handicap et de la santé $(\mathrm{CIF})^{1}$, ait favorisé l'efficacité de la collaboration dans certains groupes. Une harmonisation des enseignements, voire leur mutualisation au sein des trois filières, sur l'utilisation de ces outils faciliterait probablement les échanges et la mise en place d'une problématique commune entre les étudiants. Ces échanges, dès la formation initiale, leur permettraient également une compréhension plus fine des problématiques de santé et de la place de chacune des professions dans les parcours de soins.

Enfin, nous avons constaté que beaucoup d'étudiants assimilent la collaboration interprofessionnelle à une mise en commun de savoirs ou de pratiques «identiques » entre les professions. La formation doit donc amener les étudiants à réfléchir à la construction d'outils communs, de cadres de références, ou à l'utilisation de ceux qui existent déjà, ce qui permettrait peut-être de limiter la crainte de chacun de se voir dérober une partie de son territoire professionnel. Le dispositif de formation proposé ne représente pas une véritable situation professionnelle, avec toutes les contraintes qu'elle engage. Par contre, le discours des étudiants rend compte de l'influence des représentations favorisée par les formations initiales dispensées en « silo ». Au cours du travail collaboratif, la professionnalité émergente des étudiants a été mise en dissension avec ce que Clot appelle « le genre professionnel» et que l'on peut définir comme la mémoire collective à l'origine d'actions automatisées au sein d'une profession. «C'est comme "un mot de passe" connu seulement de ceux qui appartiennent au même horizon

\footnotetext{
${ }^{1}$ http://apps.who.int/iris/bitstream/handle/10665/42418/9242545422_fre.pdf;jsessionid=9A413ABA2ABB4B0C AD4300D558E3F8EF?sequence=1
} 
social et professionnel.» (Clot et Faïta, 2000). Le cursus des formations de rééducation/réadaptation en deuxième et troisième année se poursuit, depuis peu, par de nombreux enseignements en «inter-filiarité » notamment au travers du service sanitaire des étudiants en santé (SSES) et des certifications en éducation thérapeutique des patients (ETP).

\section{Conclusion et perspectives}

L'horizon social des étudiants devient alors transversal à plusieurs professions. Dans ces situations, comment développer une professionnalité indispensable à l'action spécifique qu'engage une profession pour répondre au besoin d'un patient ? Comment se reconnaître dans un genre professionnel et trouver sa place dans les parcours de santé ? L'appropriation de nouveaux gestes professionnels se fait par la confrontation individuelle et répétée à de nouvelles situations et permet en définitive de se reconnaître dans un collectif (Tomás, Simonet, Clot et Fernandez, 2009). La formation interprofessionnelle permettrait-elle alors de développer des compétences collaboratives transverses ? Si cette recherche était étendue aux étudiants de troisième année peut-être aurions-nous pu observer une évolution des professionnalités engageant des pratiques davantage tournées vers l'interprofessionnalité. Le milieu de la santé est en perpétuel mouvement, les référentiels des formations initiales évoluent régulièrement, les ressources sociales se transforment impliquant des changements individuels et un remodelage des professionnalités. Une approche par le «genre professionnel » (Clot et Faïta, 2000) pourrait sûrement permettre une meilleure compréhension de la construction de la professionnalité de ces étudiants au sein d'un collectif tourné vers des pratiques collaboratives interprofessionnelles.

\section{Références bibliographiques}

Aiguier, G., Poirette, S. et Pélissier, M.-F. (2016). Accompagner l'apprentissage de la collaboration interprofessionnelle: une nécessaire gouvernance réflexive du dispositif pédagogique. Journal International de Bioéthique, 27, 91-112.

Careau, E. (2013). Processus de collaboration interprofessionnelle en santé et services sociaux. Proposition d'une grille d'observation des rencontres d'équipes interdisciplinaires. Thèse présentée à la Faculté des études supérieures postdoctorales de l'Université de Laval pour l'obtention du grade de philosophiae doctor. Québec. http://hdl.handle.net/20.500.11794/24136

Careau, E., Brière, N., Houle, N., Dumont, S., Maziade, J., Paré, L., Desaulniers, M. et Museux, A.-C. (2014). Continuum des pratiques de collaboration interprofessionnelle en santé et services sociaux - Guide explicatif. Réseau de collaboration sur les pratiques interprofessionnelles en santé et services sociaux (RCPI). https://www.rcpi.ulaval.ca/fileadmin/media/documents/Outils/Guide_explicatifContinuum_vFR_18-07-31.pdf 
Clot, Y. et Faïta, D. (2000). Genres et styles en analyse du travail. Concepts et méthodes. Travailler, 4, 7-42.

D'Amour D., Sicotte C. et Lévy R. (1999). L'action collective au sein d'équipes interprofessionnelles dans les services de santé. Sciences sociales et santé. 17 (3), 67-94. DOI : 10.3406/sosan.1999.1468

Dubar, C. (2015). La socialisation comme construction sociale de la réalité. Dans C. Dubar, La socialisation : Construction des identités sociales et professionnelles (p. 79-102). Armand Colin.

Fiquet, L., Huge, S., Annezo, F. (2015), Une formation inter professionnelle pour apprendre à travailler ensemble. La perception des étudiants en santé. Pédagogie Médicale, 16, 105-117. EDP Sciences / SIFEM. DOI : 10.1051/pmed/2015018

Gachoud, D., Gallant, S., Lucarelli, L. et Oberhauser, N. (2017). Éducation interprofessionnelle et pratique collaborative, le modèle de Lausanne. Éditions Médecine et Hygiène.

Henri, F. et Lundgren-Cayrol, K. (2001). Apprentissage collaboratif à distance. Presses universitaires du Québec.

Jorro, A. (2011). Reconnaître la professionnalité émergente. Dans A. Jorro et J.-M. De Ketele La professionnalité émergente : quelle reconnaissance (p. 7-16). De Boeck Supérieur.

Mourey, F. (2005). Le rééducateur. Dans M. Aubert, D. Manière, F. Mourey et S. Outata, Interprofessionnalité en gérontologie (p. 186-192). ÉRÈS, Pratiques gérontologiques.

Oandasa, I., Baker, R-G., Barker K., Bosco, C., D’amour, D., Jones, L., Kimpton, S., LemieuxCharles, L., Nasmith, L., San Martine Rodriguez, L., Tepper, J. et Way, D., (2006). Le travail en équipe dans les services de santé : promouvoir un travail en équipe efficace dans les services de santé au Canada. Fondation canadienne de la recherche sur les services de santé.

Tomás, J., Simonet, P., Clot, Y. et Fernandez, G. (2009). Le corps : l'œuvre du collectif de travail. Corps, 6(1), 23-30. 


\section{Annexe 1}

Grilles d'observations directes réalisées au cours du dispositif de formation à la collaboration interprofessionnelle.

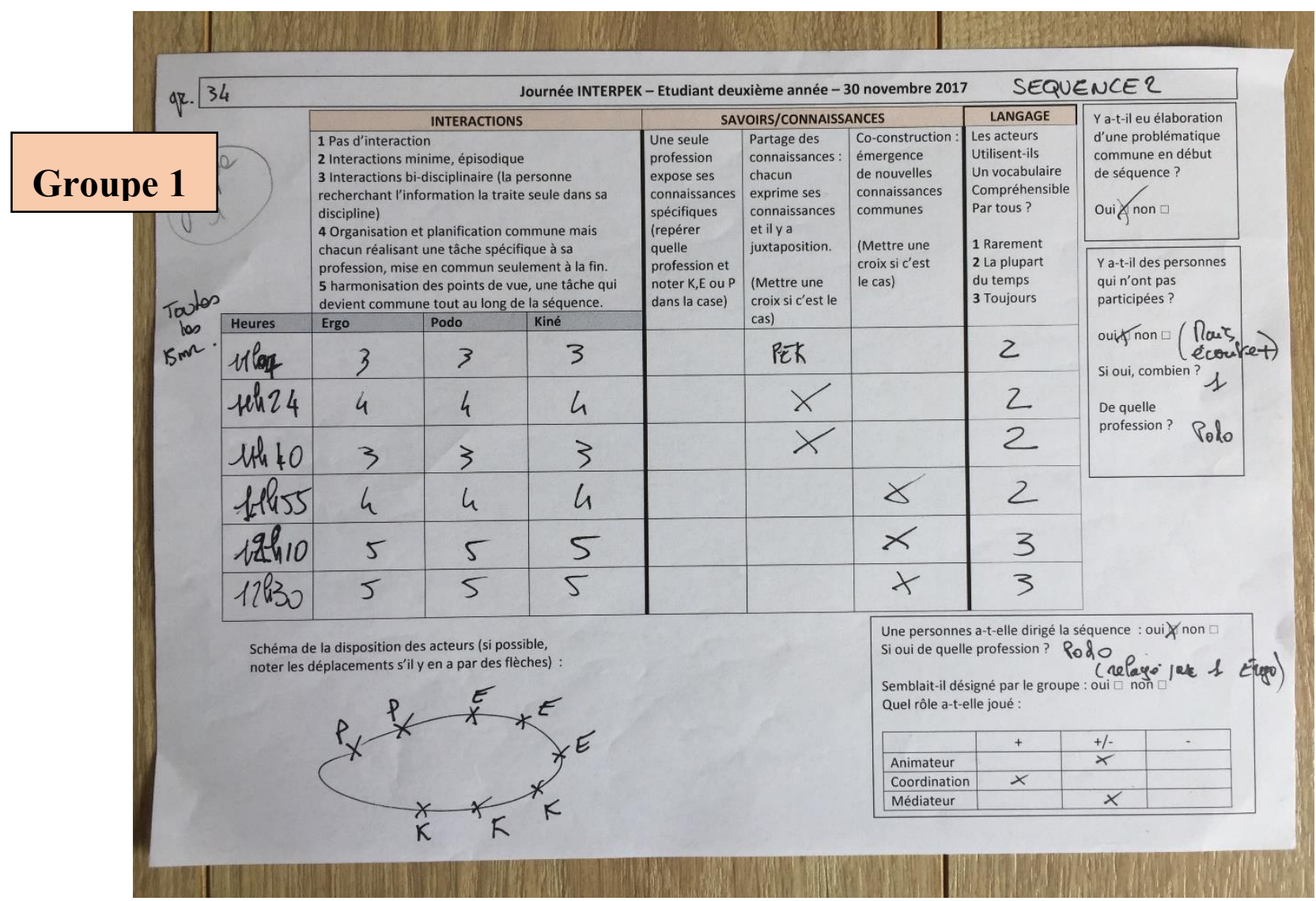

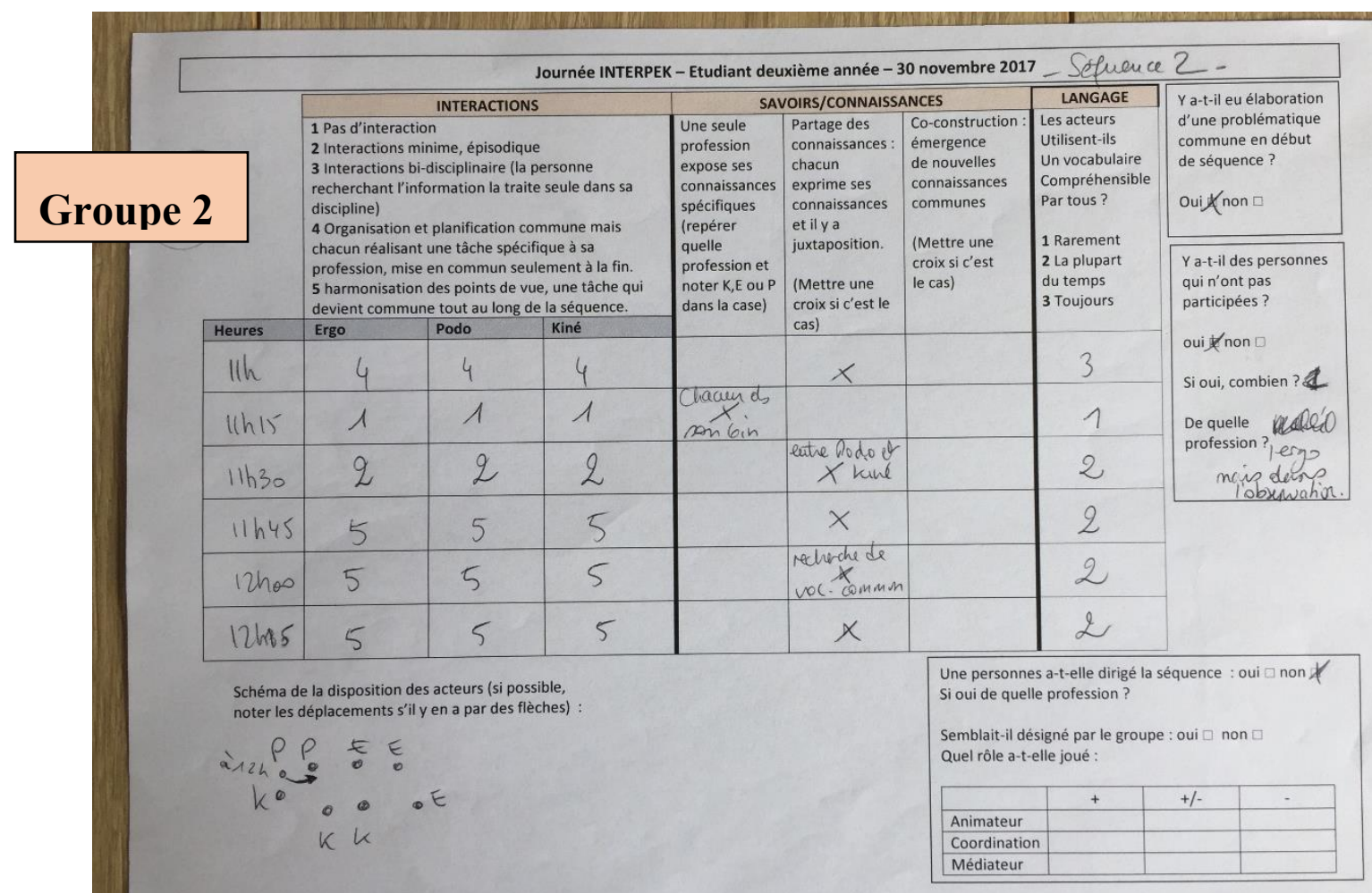




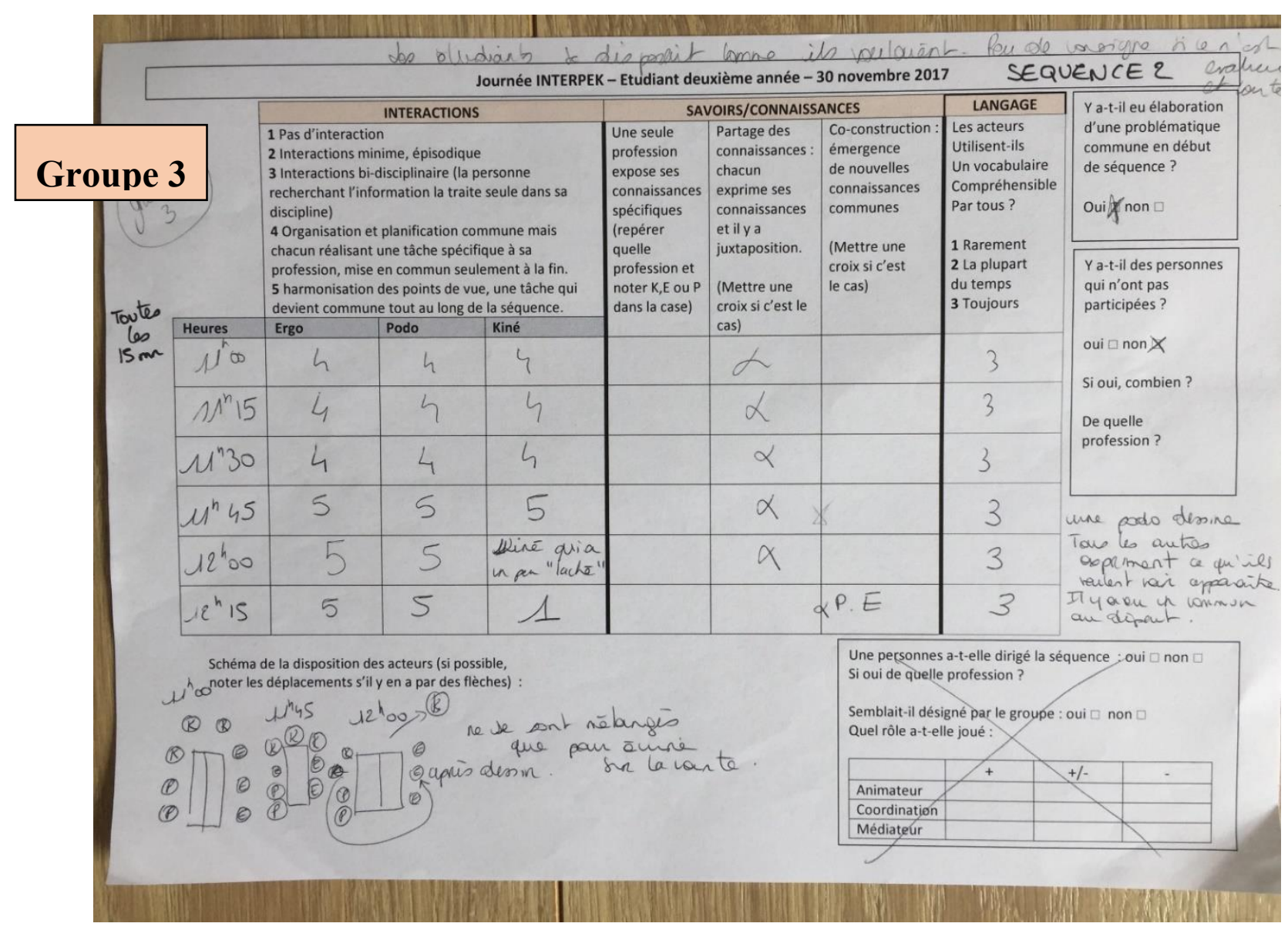

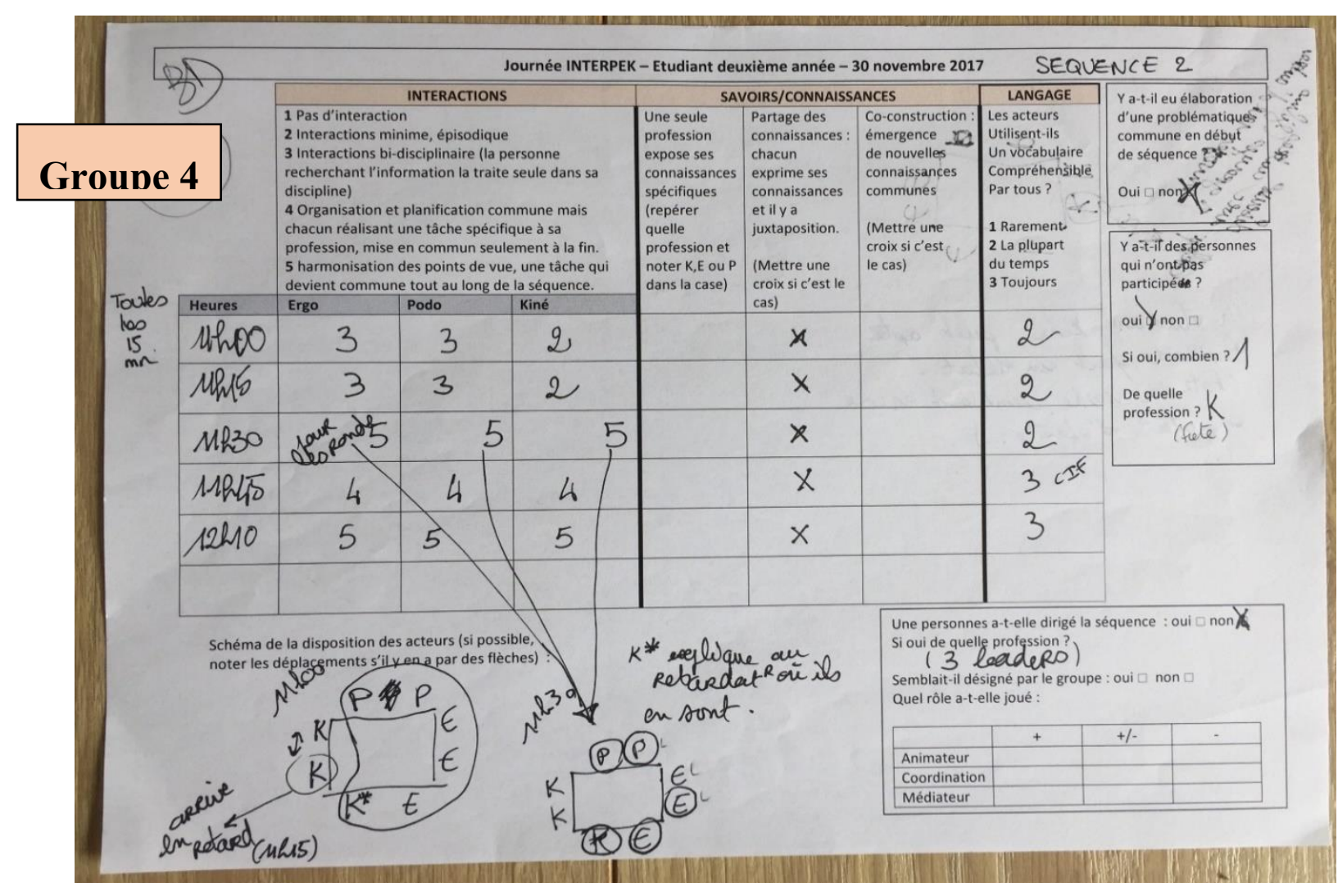




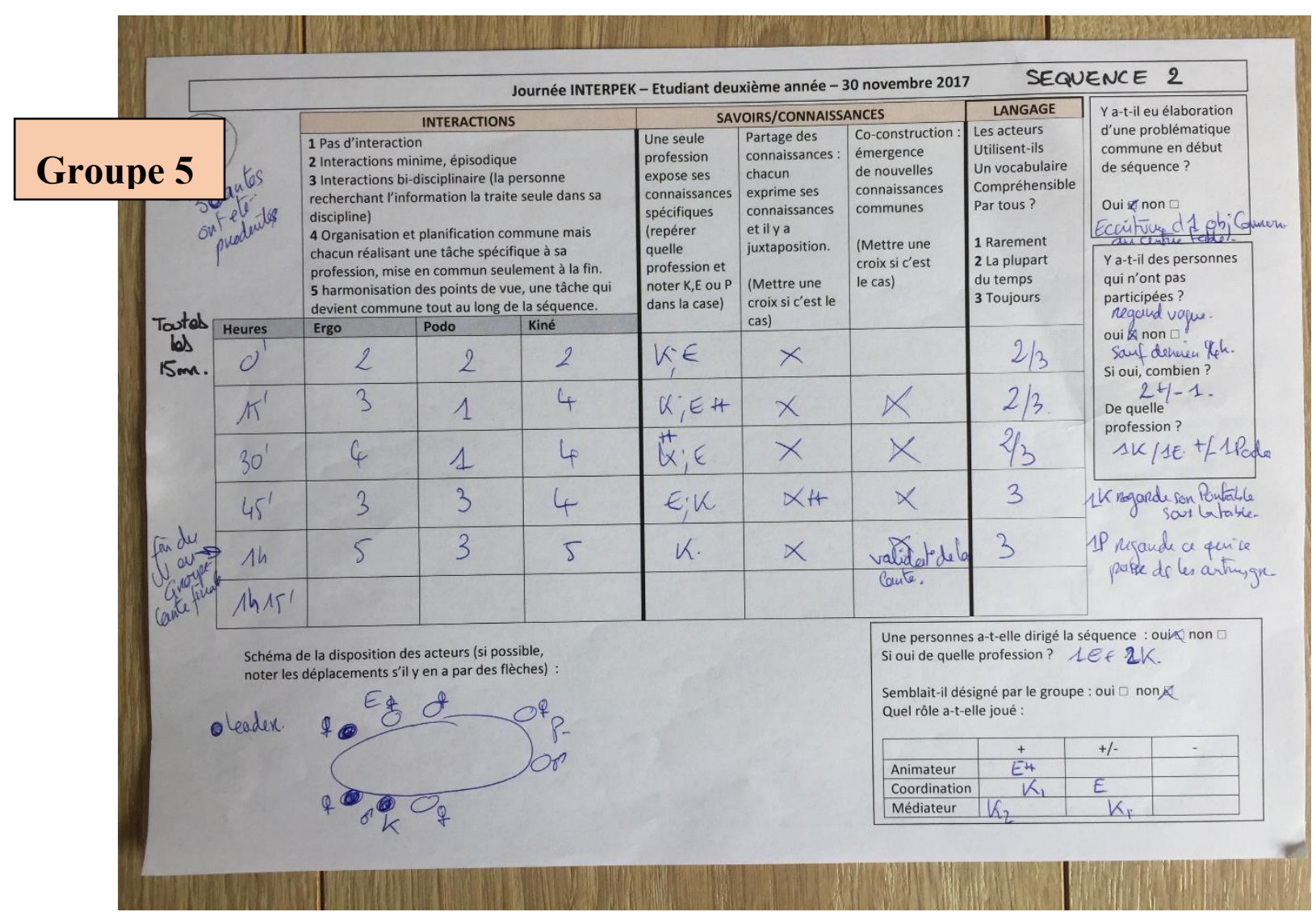




\section{Annexe 2}

\section{Guide d'entretien - étudiants IFPEK- 2ème année}

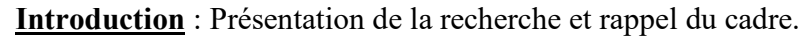

Le tutoiement est privilégié dans ce questionnaire car il est utilisé, au quotidien, avec les étudiants au sein de l'institut.

Choix professionnel

Peux-tu m'expliquer pourquoi tu as choisi cette profession?

\section{Pratiques collaboratives}

Qu'est-ce cela implique pour toi de travailler avec d'autres professionnels ?

\section{Connaissances des autres}

Peux-tu me décrire la profession des autres étudiants de l'IFPEK ?

$\sim$ Penses-tu que ce soit important de les côtoyer? Pourquoi ?

Pour toi, qu'est-ce qu'il serait important de partager avec eux dans la formation ?

Penses-tu qu'il existe une hiérarchie entre les professions médico-sociales ?

Relance :

D'où vient-elle?

Quelles conséquences cela pourrait avoir pour travailler ensemble?

\section{Retour d'expérience sur les pratiques}

Peux-tu me parler de la journée « InterPEK » et plus précisément de la séquence du matin, au cours de laquelle vous avez eu à répondre à une problématique de santé complexe entre étudiants de filières différentes ? (Support visuel : carte mentale)

Comment as-tu communiqué/échangé avec les autres professions ?

$\sim$ Quelle organisation avez-vous mis en place pour répondre au besoin du patient ?

$\sim \quad$ Quel rôle as-tu eu pendant ce travail ?

$\sim$ Peux-tu me décrire la participation de chacun?

Est-ce que tu penses que travailler ensemble a été nécessaire pour répondre à la problématique du patient ?

\section{Développement professionnel}

Qu'est-ce que cette expérience t'a apporté?

\section{Pratiques envisagées}

Suite à cette expérience, devant un patient qui a une problématique de santé complexe, que mettrais-tu en place pour faciliter le «travail ensemble»? 


\section{Analyse et classement des cartes mentales}

\section{1 cartes mentales se rapprochant des composantes des pratiques de soins et de services partagés}

La présence des trois filières sur ces cartes suppose qu'il y ait eu une bonne cohésion au sein du groupe et un engagement de la part de chaque profession pour construire un parcours de soins personnalisé pour la personne. On y retrouve également les autres composantes des pratiques de soins et de services partagés. Soit, des évaluations proposées en lien avec la problématique commune; des spécifictés par profession, interdépendantes, autour du patient et qui ont donc probablement necessité un échange de savoirs disciplinaires que chacun des acteurs s'est approprié pour réaliser un ensemble cohérent.

La carte $\mathrm{n}^{\circ} 11$ semble avoir été construite en collaboration entre deux filières de formation uniquement.

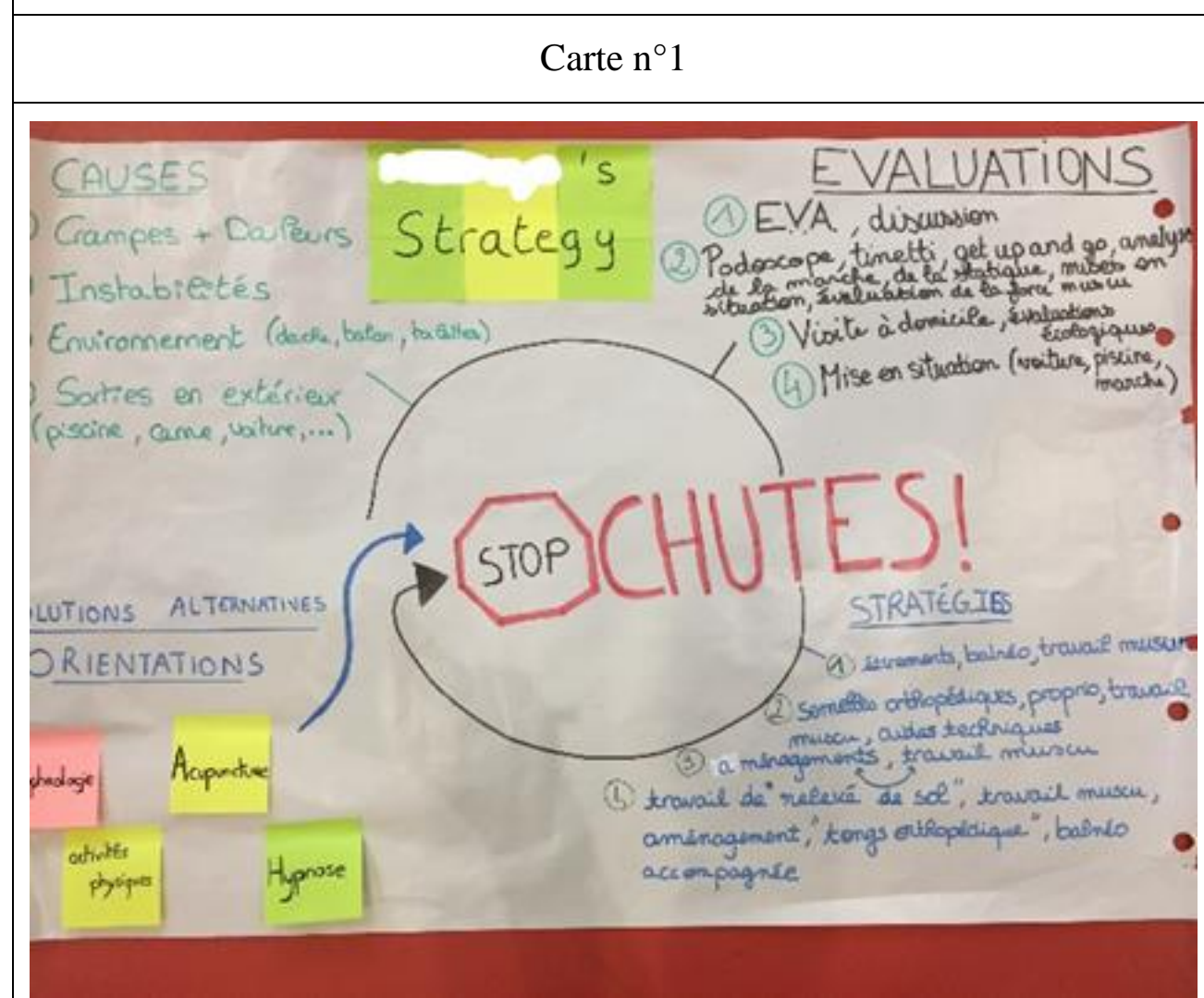

$\mathbf{E} 3 * \mathbf{E} \quad$ (u) $\quad$ Alors euh... déjà on a tout mis en commun nos, toutes les évaluations que chaque profession pouvait faire on s'est très vite rendu compte qu'on n'en faisait fin... on faisait plus ou moins les mêmes et il y avait juste quelques évaluation qui étaient spécifiques à chaque métier mais sinon on faisait globalement les mêmes. Donc, on est parti de ça. Ensuite on a vu justement bah par rapport aux causes et aux stratégies qu'on avait aussi les mêmes. Que on prenait la personne tous en globalité et que même si les kinés c'étaient plus fonctionnel, les podos plus la posture bah, on avait le même objectif, après c'est venu tous ensemble.

$\mathbf{E} 3 * \mathbf{E} \quad$ (d2) Alors du coup, on a fait l'escargot qui revenait à l'objectif principal qui était de stopper les chutes. C'était l'élément le plus risqué. C'était ce qu'il voulait arrêter. Euh, du coup, on es parti, on a regroupé les causes en quatre catégories. De ces quatre catégories on a vu les évaluations qu'on pouvait faire pour évaluer tous les risques à chaque fois.

$\mathbf{E} 2 * \mathbf{E} \quad$ (p1) Oui, on a tous trouvé notre place. Il n'y a pas eu de personne à côté qui ne faisait rien ou qui en faisait trop. En tout cas à six, on était bien. D'après moi. Tout le monde a participé, de la même manière ? Ouais.

\section{Carte $\mathrm{n}^{\circ} 2$}




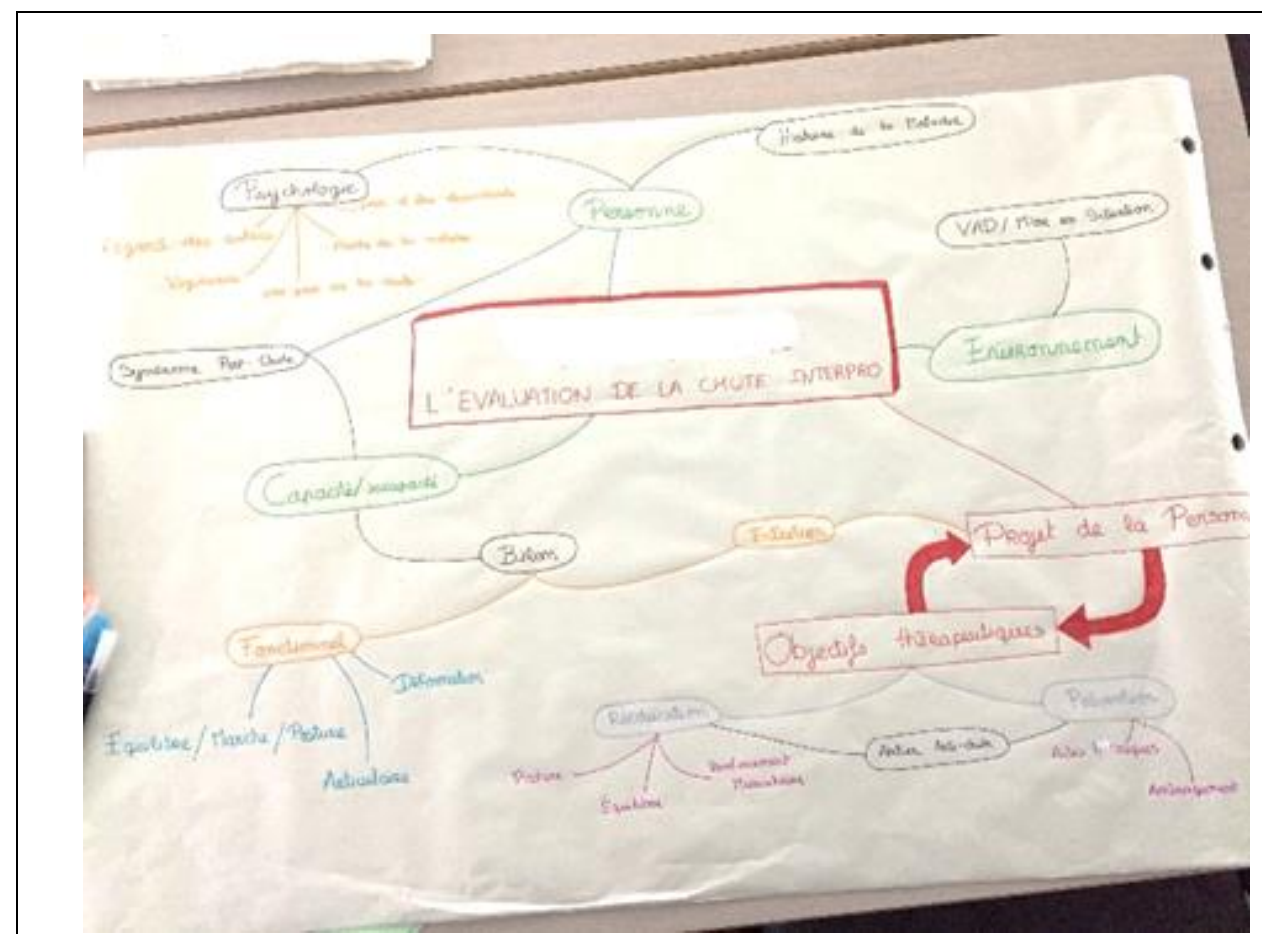

Carte $\mathrm{n}^{\circ} 3$

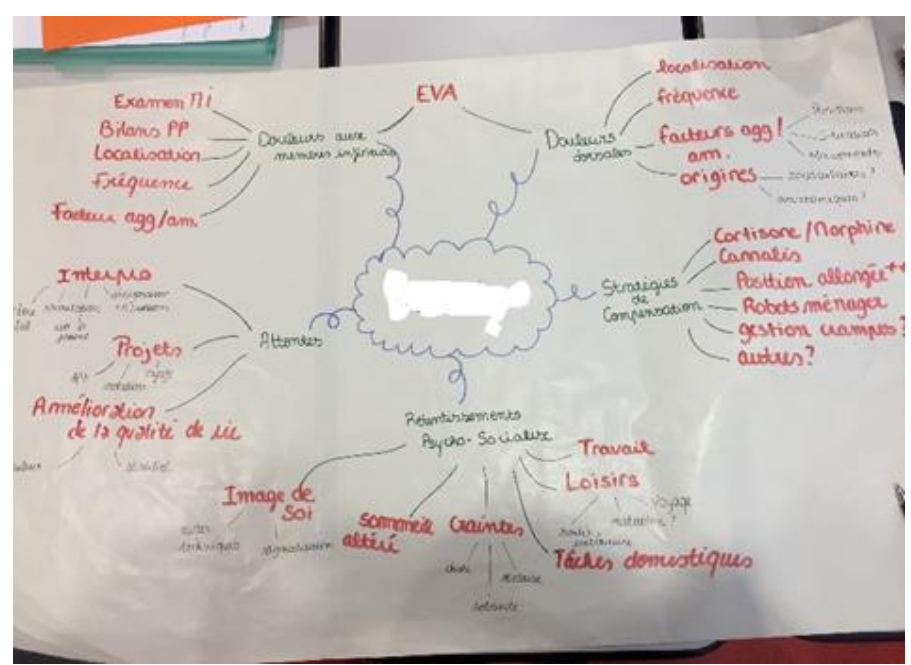

Carte $n^{\circ} 4$
E6*K (u)

Donc après, ce qui était surtout important pour notre fiche, c'était le côté bilan. Donc, pareil on a remarqué qu'à chaque fois il y avait un côté plus entretien avec la personne est un côté plutôt fonctionnel. Euh et ce qui ressortait aussi dans les trois professions c'était qu'à la fin du bilan euh... On montait une sorte... un projet. Un projet pour la personne.

E6*K (t) Bah, c'est pas vraiment spécifique au kiné ou spécifique à l'ergo mais on a remarqué en tout cas que quand on va découvrir euh... une personne on va s'intéresser à la personne en ellemême, à son environnement et à ses capacités voilà. Donc il y avait ces trois points là qui était vraiment important pour chaque profession. Donc après, on a essayé de détailler un peu sur la personne...

E8*P (u) Et même si on se sentait pas impliqué sur le coup, au final chacun à sa pierre à donner à l'édifice et c'était intéressant d'échanger et vraiment ces questions de points de vue c'est intéressant comment par exemple le kiné va vraiment se concentrer sur ces douleurs, comment on va lui faire des exercices, où parce qu'il ne veut pas trop sortir euh... L'ergo qui essaie de tout adapter pour que ce soit plus facile pour lui, nous qui essayons de trouver notre.... Et c'était intéressant vraiment, on rebondissait euh... C'était ouais... c'était vraiment enrichissant comme prise en charge.

E10*P (n1) Ouais voilà, bah nous par exemple on ne savait pas. Du coup fin, il y a plein de choses 


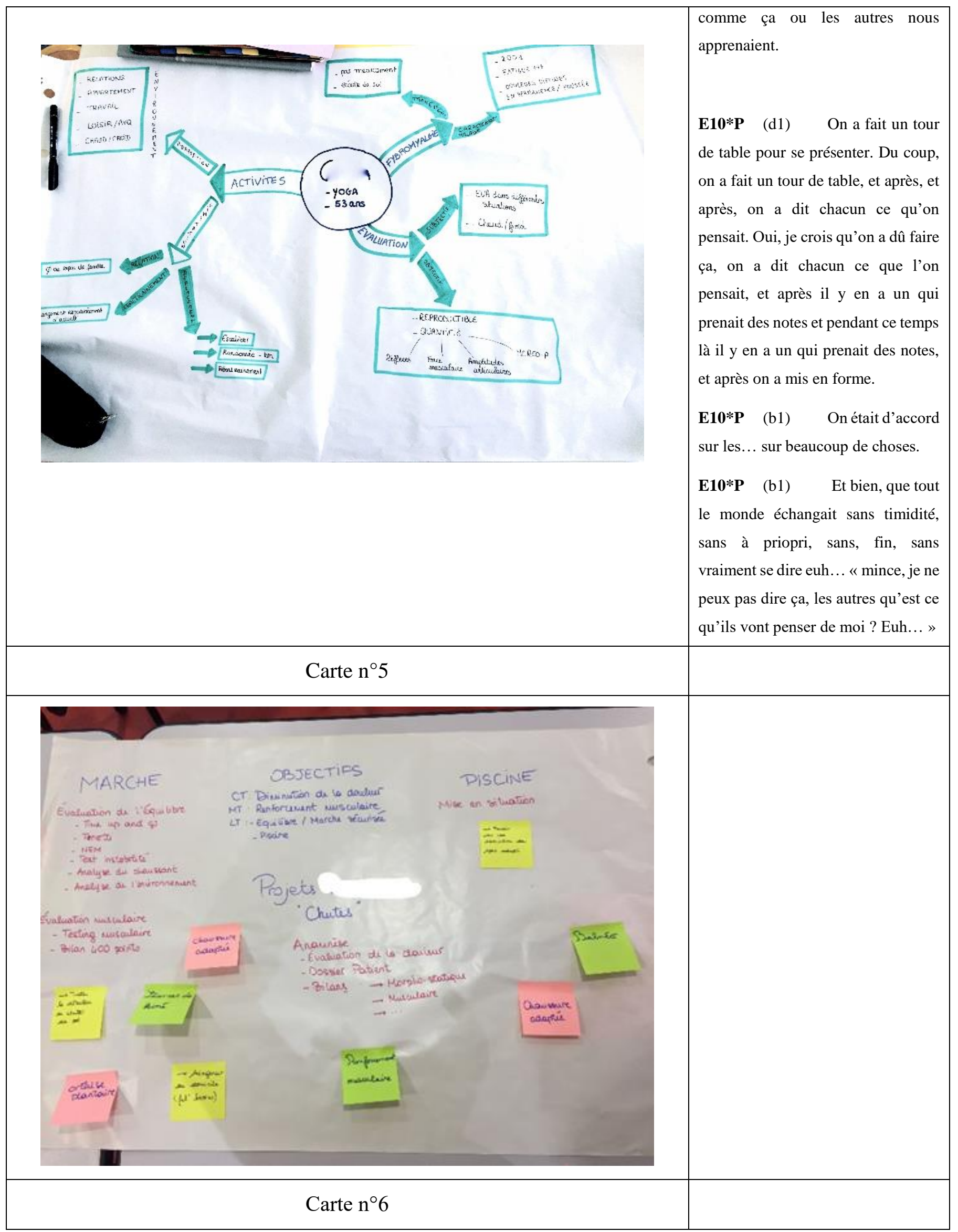




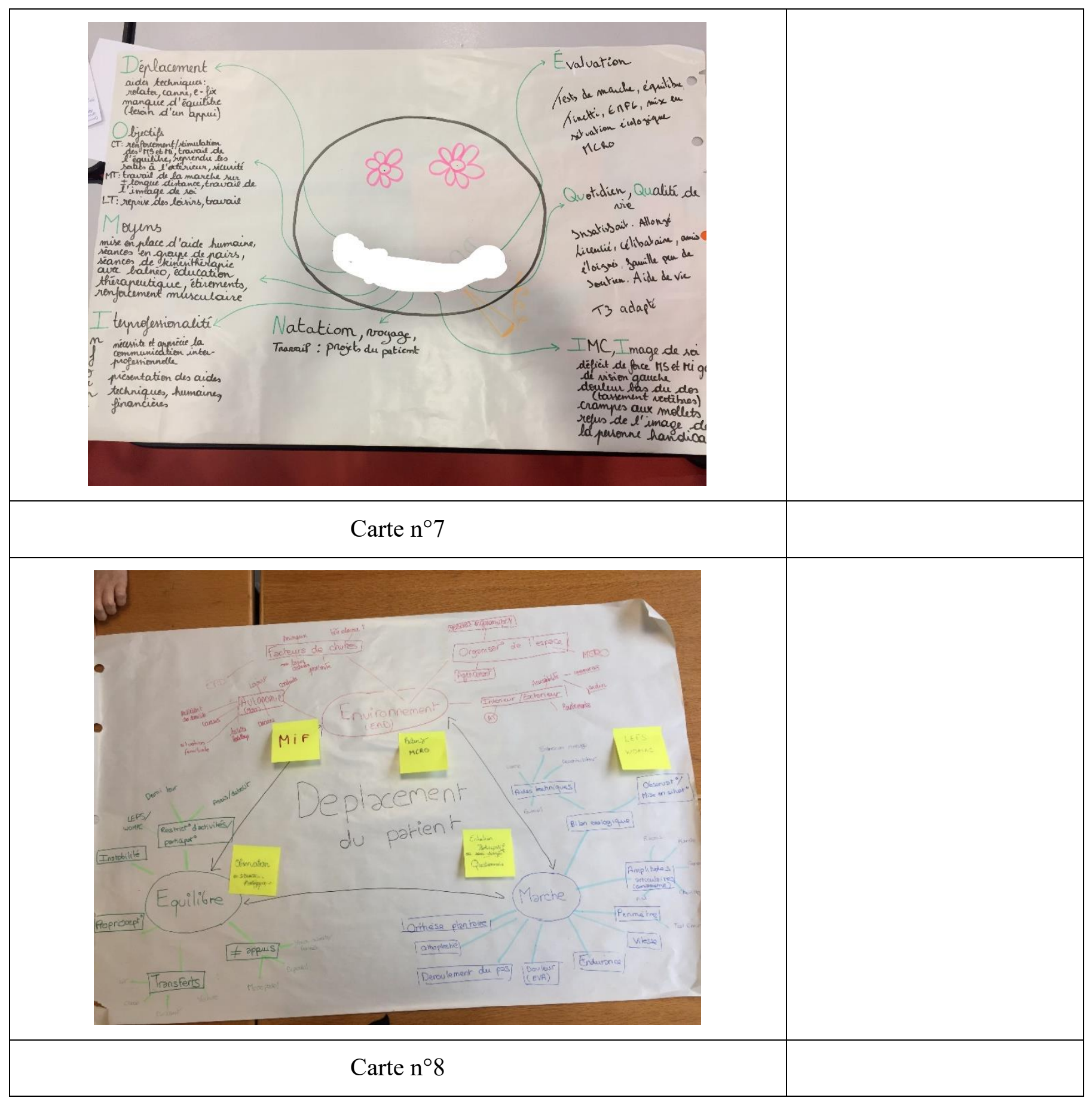




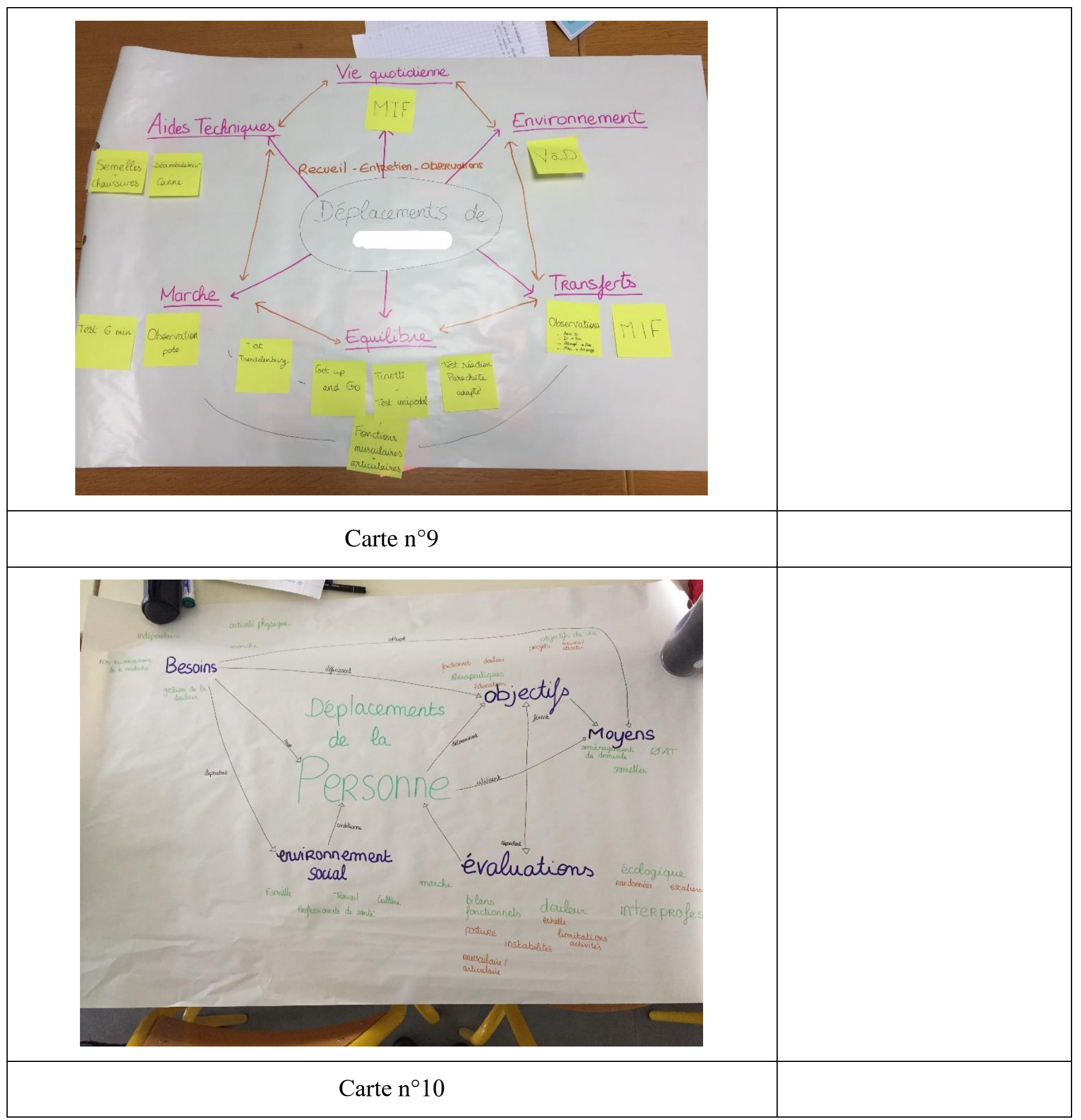




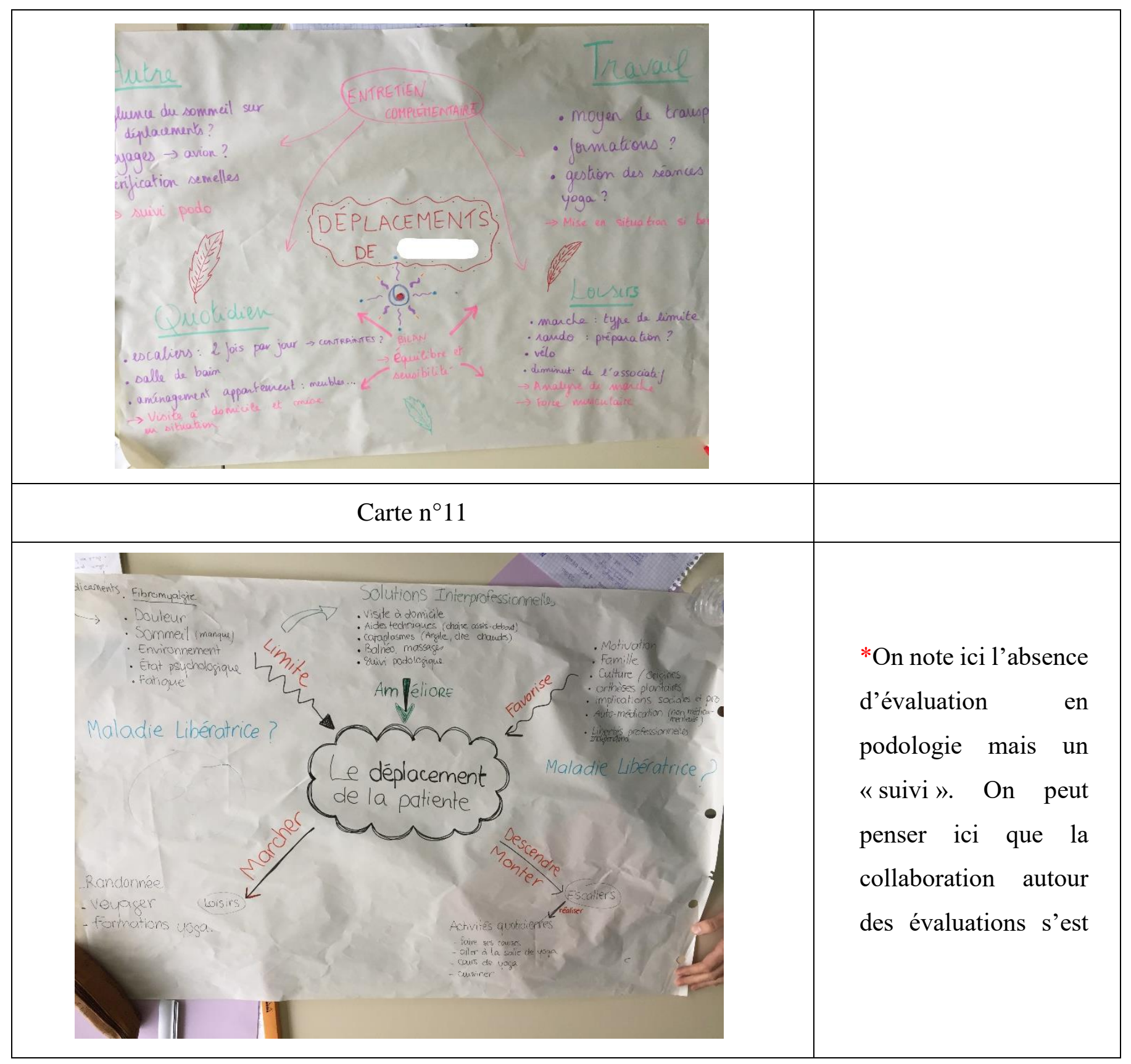




\section{$\mathbf{2}$ cartes mentales : Recueil de données commun et juxtaposition des évaluations}

Ces cartes présentent une réflexion inter-filières pour le recueil des données auprès du patient. Sur la première carte, le recueil des données est posé par rapport à la personne mais la problématique commune n'est pas mise en évidence.

Sur la deuxième carte, l'objectif commun n'est pas personnalisé par rapport au projet réel de la personne, il s'apparente à la thématique donnée en début d'exercice. Le fractionnement des évaluations et de la mise en place des soins nous fait davantage penser à de la coopération.

Pour ces deux cartes, nous observons une juxtaposition des pratiques sans qu'il y ait véritablement besoin d'échanger entre professionnels sur les actions à mettre en place le long du parcours de soins. L'interdépendance est moindre entre les professions.

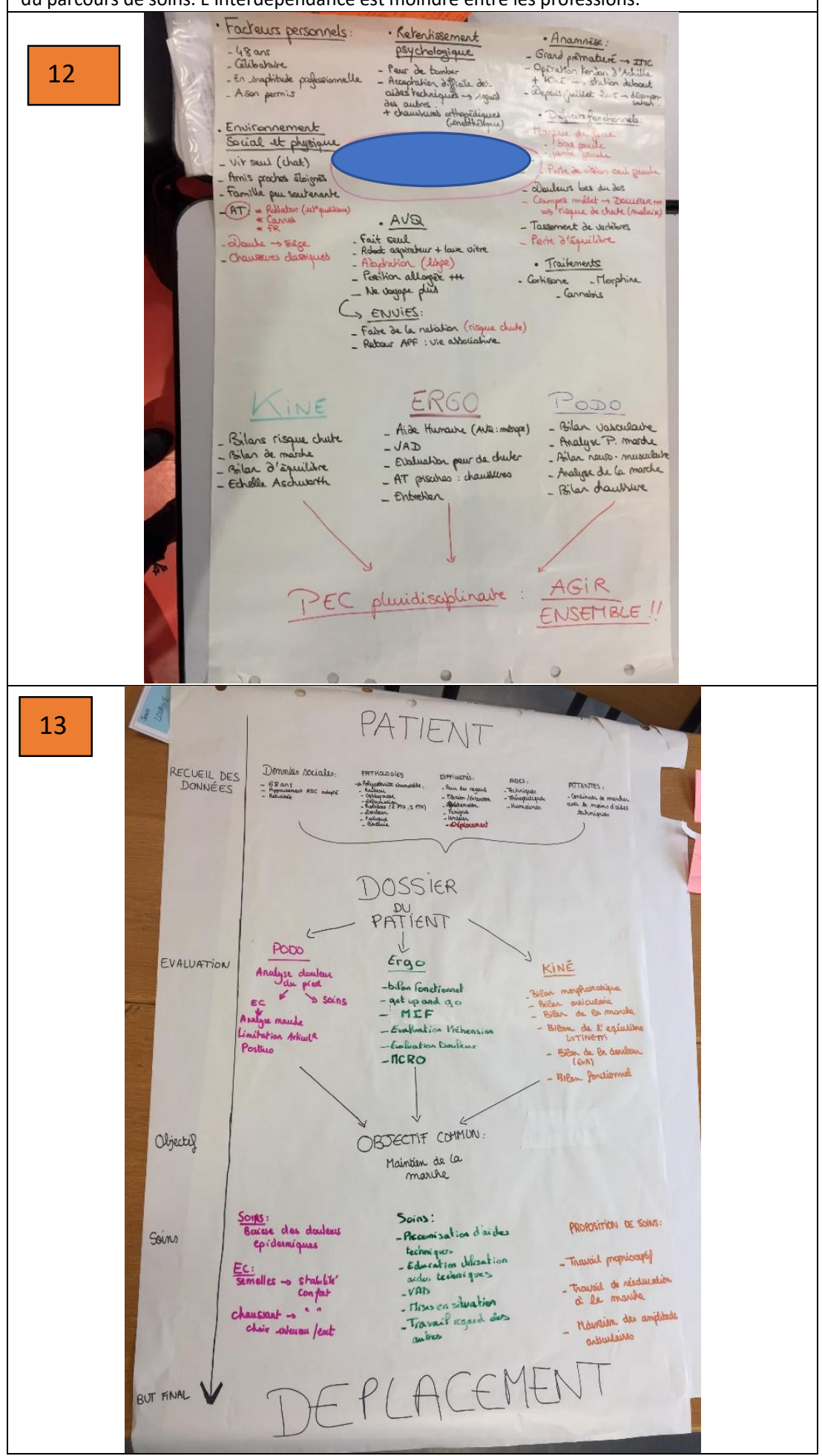




\section{$\mathbf{2}$ carte mentale : recueil des données uniquement}

Ces deux cartes présentent uniquement le profil occupationnel et l'état occupationnel de la personne. Il n'y a pas de problématique et donc pas de positionnement professionnel sur un parcours de soins. Les étudiants ont collaboré pour effectuer une présentation globale de la personne à travers une approche bio-psychosociale.

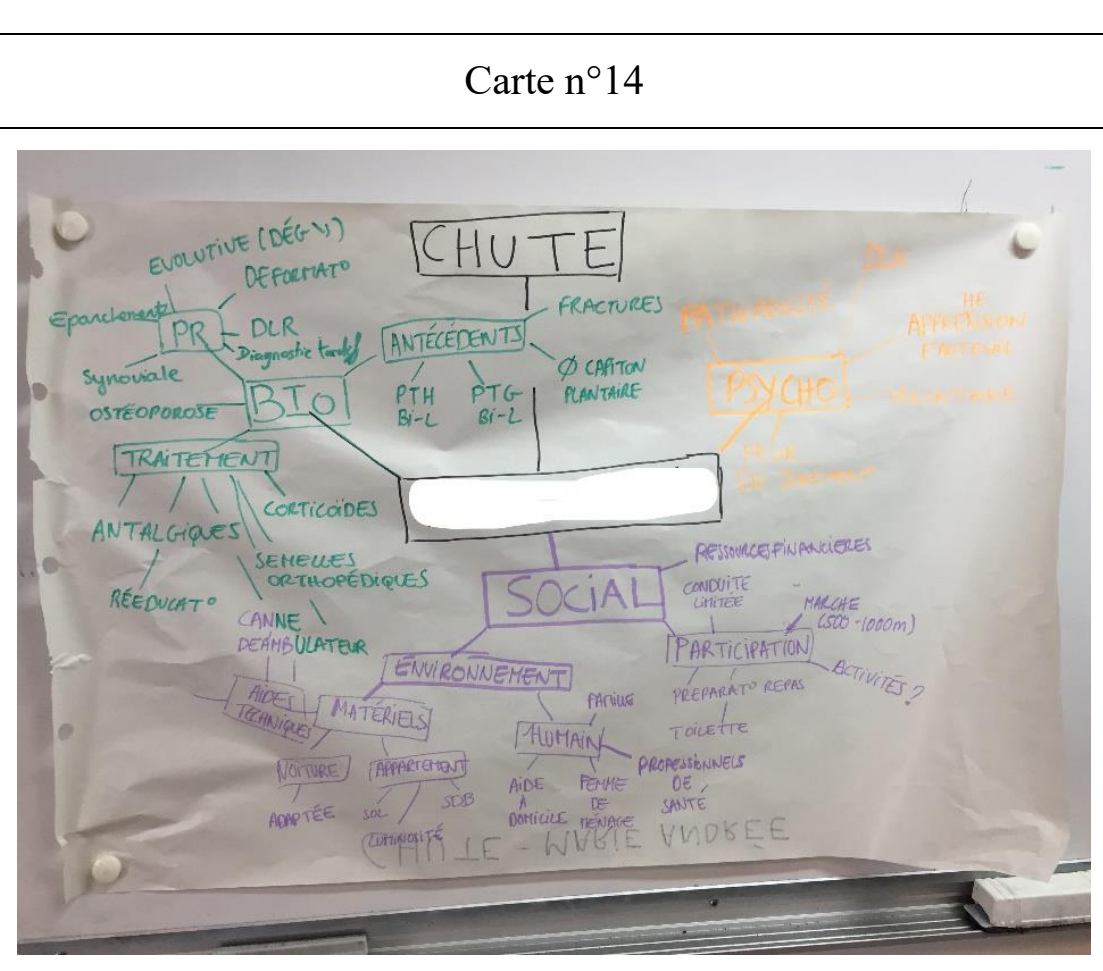

Carte ${ }^{\circ} 15$ 


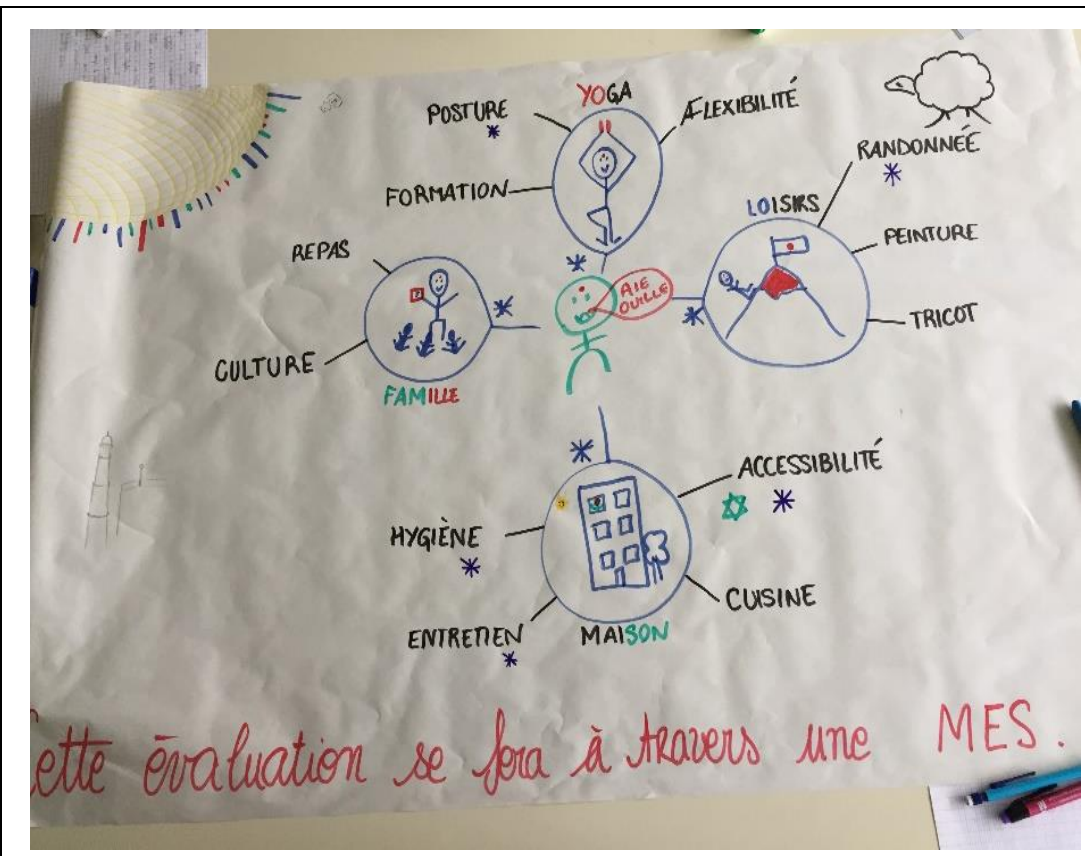

E4*E (q) Je crois que c'est ergo. On a émis l'idée de cibler vraiment ses activités principales est-ce qu'il importait pour elle en fait. Et à ce momentlà d'ailleurs on leur a expliqué vraiment c'était aussi hyper primordial dans l'ergothérapie de voir chez cette personne euh.... Qu'est-ce qui l'importe et enfin voilà... ce qui constitue son quotidien.

$\mathbf{E} 4 * \mathbf{E} \quad$ (x) Je pense qu'en fait d'après ce qu'ils me disaient euh... Les kinés et les podos... c'est... Après je sais pas peut-être qu'ils font souvent comme ça, donc du coup on fait toujours comme ça même en ergo enfin... mais... ils ont besoin de voir la personne et de faire des bilans pour voir ensuite ce qu'ils peuvent faire et donc du coup là, ils ne voyaient pas trop comment... non le fait de voir peut-être qu'on pouvait pas tout à l'instant $\mathrm{T}$ parce qu'on avait pas toutes les infos. Rien que le fait de voir son appartement et d'entendre ses plaintes, on pouvait déjà essayer d'imaginer deux ou trois trucs. Mais... après oui, c'est vrai avec plus d'informations sur la personne et sur sa maladie on aurait peut-être pu plus discuter.

$\mathbf{E} 4 * \mathbf{E} \quad$ (k) A

communiquer non. On a eu... on

s'est bien entendu. A

communiquer on a pas eu de mal.

C'était plutôt à comprendre la place de chacun. 


\begin{tabular}{|c|c|}
\hline \multicolumn{2}{|c|}{$\begin{array}{l}1 \text { carte : évaluation commune juxtaposée mais pas de problématique commune ni } \\
\text { d'individualisation du parcours }\end{array}$} \\
\hline $\begin{array}{l}\text { Cette carte présente des évaluations spécifiques très générales et juxtaposées. Nous } \\
\text { n'observons pas de problématique commune et aucune analyse, ni des besoins, ni du } \\
\text { projet de la personne. L'interdépendance entre les filières est absente ainsi que la } \\
\text { proposition d'évaluation interprofessionnelle. }\end{array}$ & \\
\hline Carte $n^{\circ} 16$ & \\
\hline 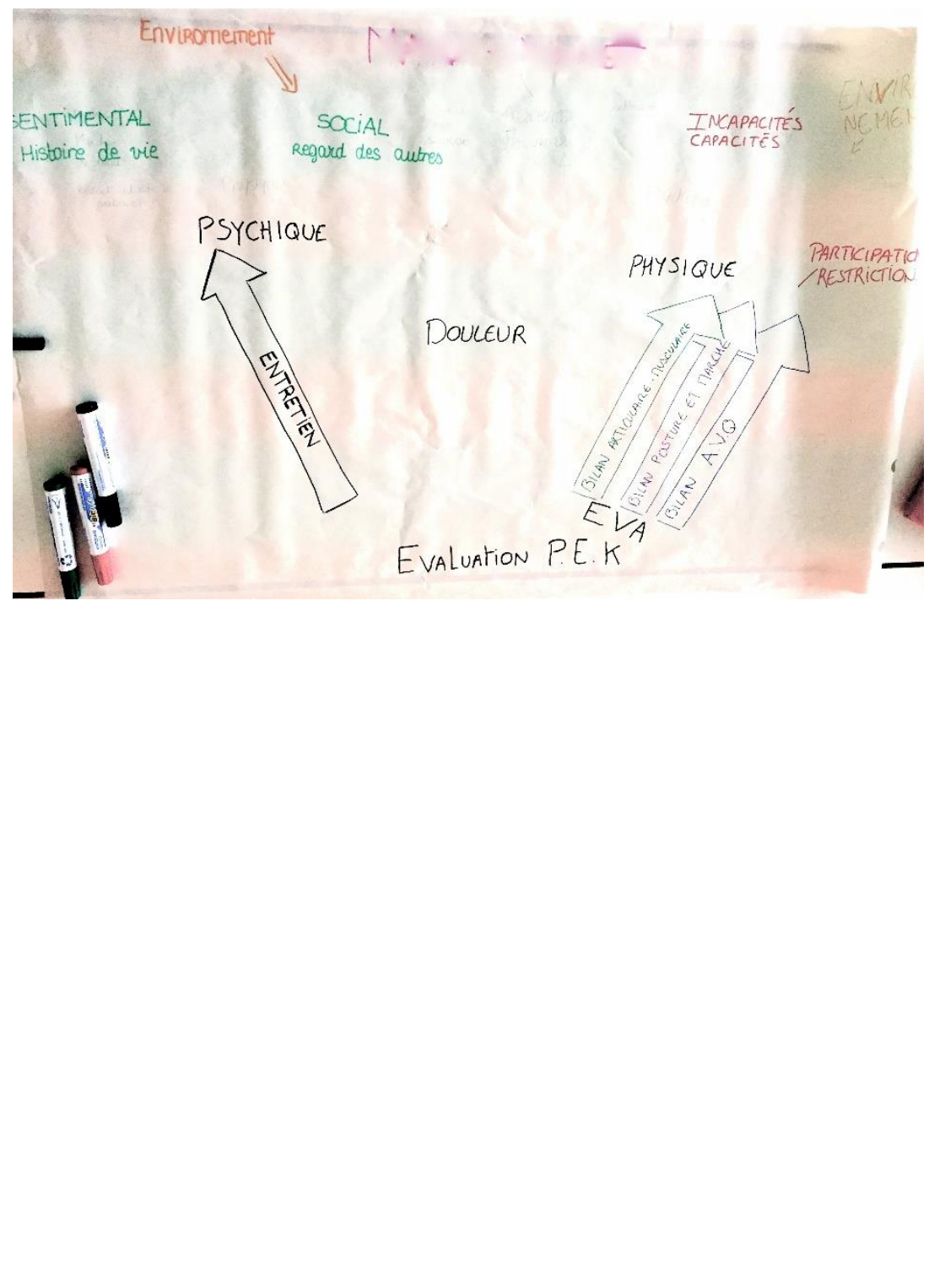 & $\begin{array}{l}\text { E5*K (el) Il me semble } \\
\text { qu'on n'a pas été jusque-là, il me } \\
\text { semble qu'on a juste évoqué le fait } \\
\text { que enfin, à l'oral on a dit les } \\
\text { différents bilans que chacun avait et } \\
\text { tout ça. Mais je ne suis pas sûre que } \\
\text { l'on a été enfin jusqu'à vraiment } \\
\text { détailler. } \\
\text { E5*K (x) Ouais, on est parti sur } \\
\text { la CIF, et en fait à partir de là on est } \\
\text { parti aussi sur le bilan. Et on se } \\
\text { disait: bah nous en kiné on a des } \\
\text { bilans, ah bah, pareil chez les podos, } \\
\text { pareil chez les ergo et fin... } \\
\text { découvrir en fait, que l'on utilisait les } \\
\text { mêmes bilans, parfois les mêmes } \\
\text { outils, donc ce seraient plutôt des } \\
\text { questionnaires, des échelles de vie et } \\
\text { tout ça. Bah, avec les ergos, parce } \\
\text { que avec le p... si, aussi avec les } \\
\text { podos. Je ne vais pas dire de bêtise } \\
\text { mais, mais il me semble que les } \\
\text { podos aussi en utilise un petit peu } \\
\text { aussi enfin voilà. On a découvert } \\
\text { qu'on utilisait des outils en commun } \\
\text { et puis que chacun avait des outils } \\
\text { plus spécifiques quoi. Mais, non, une } \\
\text { bonne dynamique dès le début fin... } \\
\text { E5*K (rl) Chaque } \\
\text { professionnel a apporté sa pierre } \\
\text { pour construire quelquechose. }\end{array}$ \\
\hline
\end{tabular}




\section{1 carte : interdépendance, individualisation}

Cette carte montre l'interdépendance entre les professions. Elle est réalisée sur les besoins propres à la personne répartis en fonction des filières : une partie spécifique et une mise en commun. Un échange disciplinaire a donc dû avoir lieu sur les champs de pratiques possibles par chacun. La problématique commune n'est pas clairement indiquée, les évaluations sont mélangées avec des propositions de moyens. Les liens entre les actions proposées ne sont pas visibles.

\section{Carte $n^{\circ} 17$}

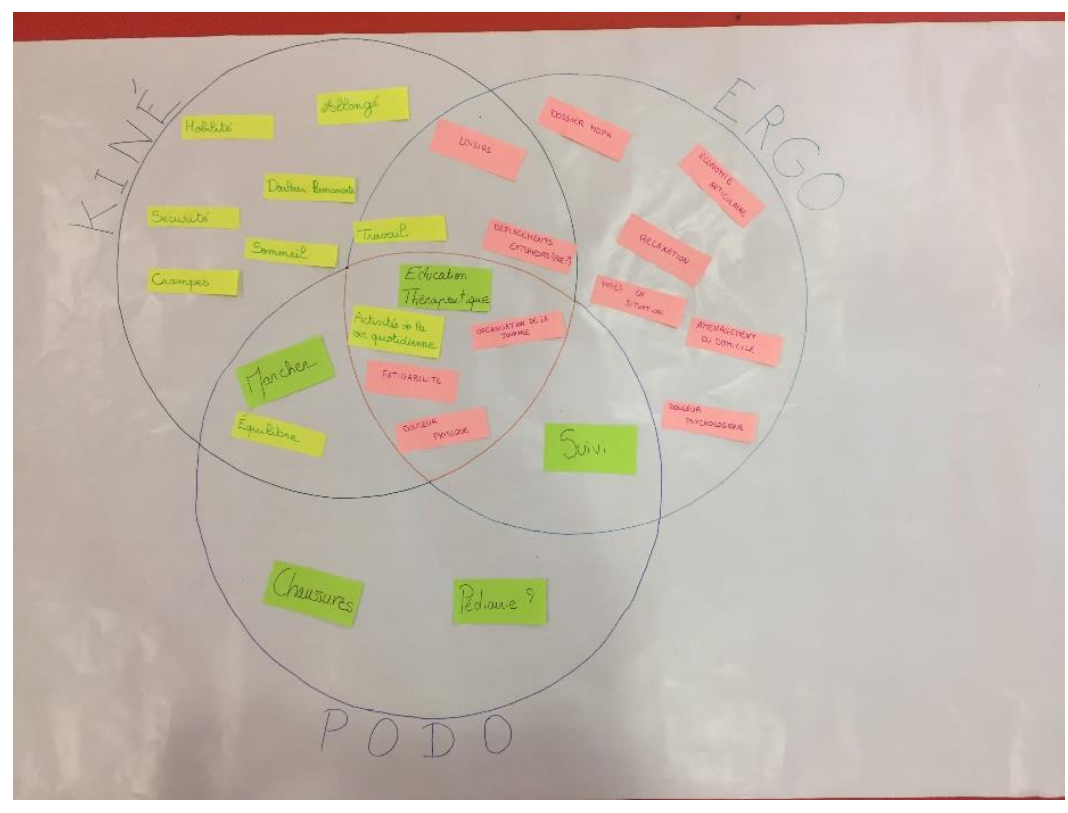

\section{1 carte : interdépendance}

Nous observons ici la représentation de l'interdépendance entre les acteurs, autour d'une problématique commune partielle, représentant une toute petite partie de la situation de handicap de la personne. Les besoins sont cependant très succincts, les projets personnels n'apparaissent pas. Les étudiants ne proposent aucune évaluation probablement par manque de mise en commun d'information sur le patient. Les échanges semblent ne pas avoir été assez riches pour l'individualisation et la proposition d'un parcours évaluatif. 


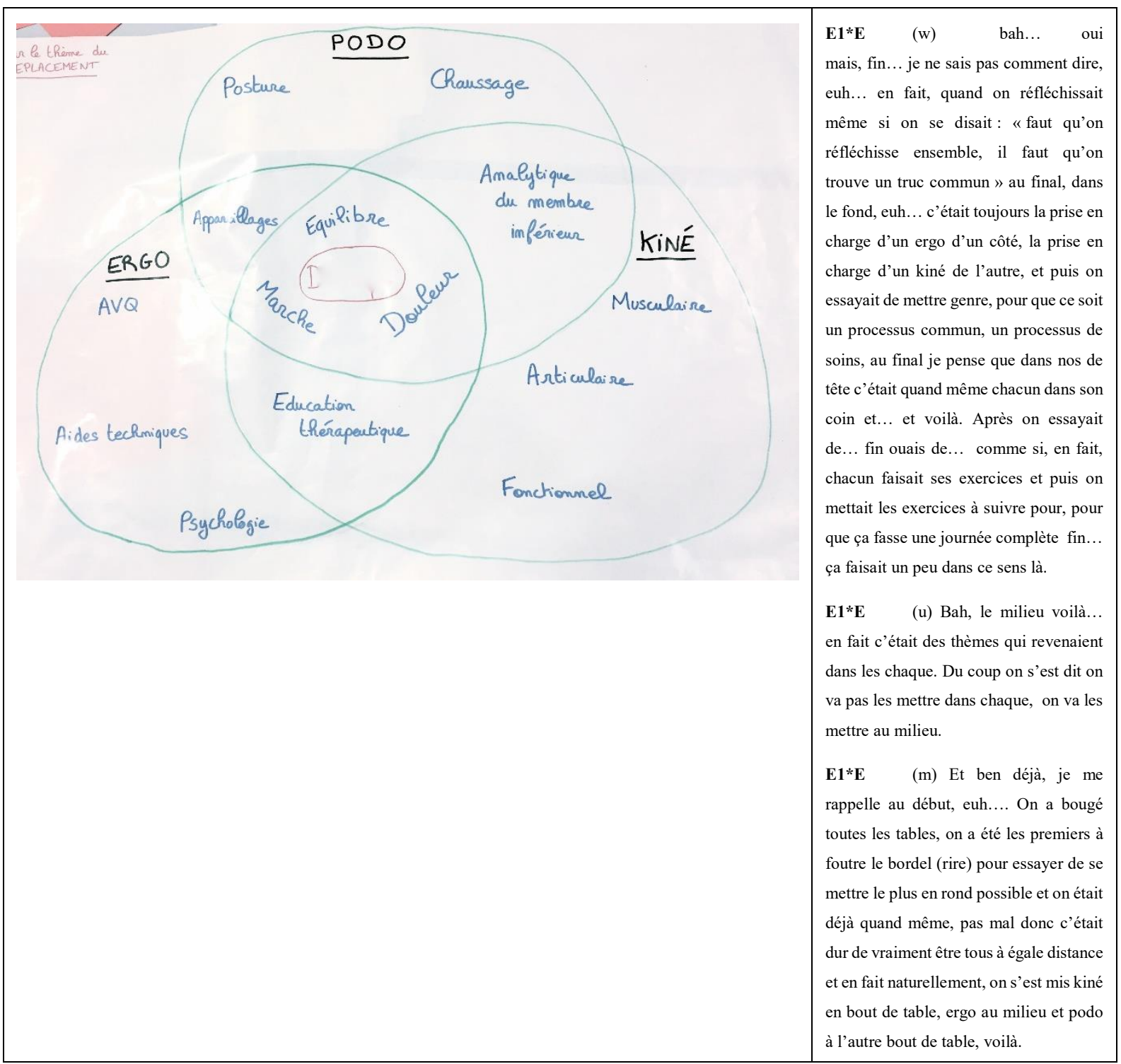

Deux cartes difficiles à mettre en lien avec les composantes des pratiques de soins et de services partagés.

Ces travaux nous ont été très difficiles à analyser, nous n'observons aucune des composantes des pratiques de soins et de services partagés.

Cartes n ${ }^{\circ} 19$ et 20 


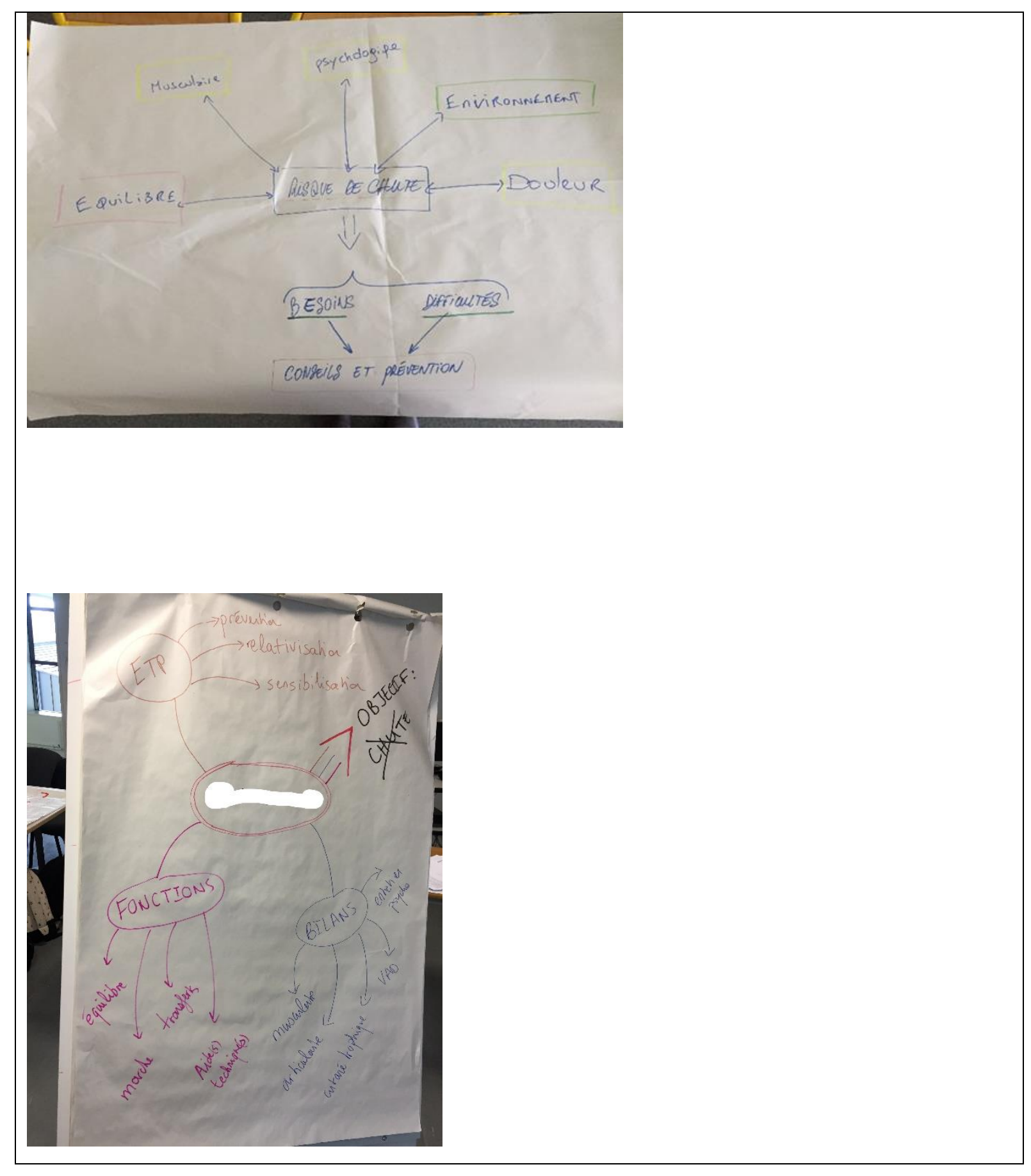

